\title{
Multiplier Ideals in Two-Dimensional Local Rings with Rational Singularities
}

\author{
Maria Alberich-CARRAMiñana, \\ Josep Àlvarez Montaner, \& Ferran Dachs-Cadefau
}

\begin{abstract}
The aim of this paper is to study jumping numbers and multiplier ideals of any ideal in a two-dimensional local ring with a rational singularity. In particular, we reveal which information encoded in a multiplier ideal determines the next jumping number. This leads to an algorithm to compute sequentially the jumping numbers and the whole chain of multiplier ideals in any desired range. As a consequence of our method, we develop the notion of jumping divisor that allows us to describe the jump between two consecutive multiplier ideals. In particular, we find a unique minimal jumping divisor that is studied extensively.
\end{abstract}

\section{Introduction}

Let $X$ be a complex algebraic variety with mild singularities, and $\mathcal{O}_{X, O}$ the local ring of a point $O \in X$. To any ideal $\mathfrak{a} \subseteq \mathcal{O}_{X, O}$ we may associate a family of $\mathrm{mul}$ tiplier ideals $\mathcal{J}\left(\mathfrak{a}^{\lambda}\right)$ parameterized by positive rational numbers $\lambda \in \mathbb{Q}_{>0}$. Indeed, they form a nested sequence of ideals

$$
\mathcal{O}_{X, O} \supseteq \mathcal{J}\left(\mathfrak{a}^{\lambda_{1}}\right) \supseteq \mathcal{J}\left(\mathfrak{a}^{\lambda_{2}}\right) \supseteq \cdots \supseteq \mathcal{J}\left(\mathfrak{a}^{\lambda_{i}}\right) \supseteq \cdots,
$$

and the rational numbers $0<\lambda_{1}<\lambda_{2}<\cdots$ where the multiplier ideals change are called jumping numbers. The first jumping number $\lambda_{1}$ is also known as the log-canonical threshold. Multiplier ideals and their associated jumping numbers have proven to be a powerful tool to understand the geometry of singularities. They are defined using a log-resolution of the pair $(X, \mathfrak{a})$. In fact, smaller or more dense jumping numbers can be thought to correspond to "worse" singularities.

The aim of this paper is to present a new approach to the understanding of multiplier ideals and jumping numbers of any ideal $\mathfrak{a}$ in the local ring $\mathcal{O}_{X, O}$ of a complex surface $X$ having at worst a rational singularity at $O$. This is a case, especially where $X$ is smooth, that has received a lot of attention in recent years because of the interesting properties these invariants satisfy (see the works of

Received December 22, 2014. Revision received January 22, 2016.

All three authors were partially supported by Generalitat de Catalunya 2014 SGR-634 project and Spanish Ministerio de Economía y Competitividad MTM2015-69135-P. FDC is also supported by the KU Leuven grant OT/11/069. MAC is also with the Institut de Robòtica i Informàtica Industrial (CSIC-UPC) and the Barcelona Graduate School of Mathematics (BGSMath). 
Favre and Jonsson [8; 9], Lipman and Watanabe [20], or Tucker [24]). This is also one of the few cases where explicit computations have been done.

For simple complete ideals or irreducible plane curves in a smooth surface, Järviletho [15] and Naie [21] provide a closed formula for the set of jumping numbers in terms of some invariants of the singularity, the Zariski exponents. To give a closed formula for any general ideal is beyond the scope of this work. A formula for the log-canonical threshold already becomes quite complicated as we may see in the papers of Kuwata [16] and Galindo, Hernando, and Monserrat [10].

For the case of any ideal in a surface with a rational singularity, we must refer to the work of Tucker [25], where he gives a simple algorithm to compute the set of jumping numbers. For such a purpose, he developed the notion of divisors that (critically) contribute, building upon previous work of Smith and Thompson [23]. We may interpret jumping numbers as being parameterized by contributing divisors, and critical divisors are more economic to detect since the complete ideals they define are very close to their corresponding multiplier ideal. The algorithm that Tucker proposes uses a characterization of critical divisors that allows them to be found and consequently allows the corresponding jumping numbers to be computed.

A similar strategy is used by Hyry and Järvilehto [14], who proved that jumping numbers are parameterized by more general complete ideals. ${ }^{1}$ Moreover, they provide a combinatorial criterion to detect a suitable ideal and its corresponding jumping number.

The aim of this paper is to understand the whole change between a multiplier ideal to the next one and to reveal what information encoded in a multiplier ideal determines the next jumping number. This is done in our main result Theorem 3.5, and it gives rise to an algorithm to compute the ordered sequence of multiplier ideals in any desired range of the real line. The algorithm avoids considering candidates and computes sequentially at each step a jumping number and its associated multiplier ideal. This new algorithm improves in efficiency the computation of jumping numbers when compared with Tucker's algorithm.

Perhaps the most important contribution of our method lies in finding a divisor, which we name the minimal jumping divisor, tightly related to the aforementioned algorithm, which enables us to obtain a multiplier ideal from the previous one, and vice versa. This jumping divisor is studied, in particular, its geometric structure on the dual graph, and it is compared with the previously known critically contributing divisors.

The structure of this paper is as follows: In Section 2 we introduce the basics of the theory of multiplier ideals and some of the tools in the theory of singularities that we will need in the rest of the paper. We pay special attention to the equivalence between complete ideals and antinef divisors developed by Lipman [19] since this is the way we will present multiplier ideals. In particular, we provide

\footnotetext{
${ }^{1}$ Contributing divisors describe complete ideals nested in between consecutive multiplier ideals. The ideals considered in [14] are not necessarily nested.
} 
a new method to compute the antinef closure of any given divisor, generalizing previous versions of Casas-Alvero [5] and Reguera [22].

In Section 3 we present the main result of this paper in Theorem 3.5. It gives a generalization of a well-known formula for the log-canonical threshold and allows us to compute a jumping number from the data given by the preceding a multiplier ideal. This leads to the desired algorithm that computes sequentially the chain of multiplier ideals.

In Section 4 we develop the theory of jumping divisors that allows us to describe the whole jump between two consecutive multiplier ideals. Quite surprisingly, the algorithm we develop in Section 3 allows us to construct the unique minimal jumping divisor associated to every jumping number. It is minimal in the sense that no proper subdivisor gives the jump between consecutive multiplier ideals. Moreover, we prove in Theorem 4.11 that minimal jumping divisors are generically invariant with respect to log-resolutions of the ideal and that they satisfy some nice geometric properties when viewed in the dual graph.

Finally, in Section 5 we present the theory of jumping divisors in a more general framework that we develop using the results of Hyry and Järvilehto [14] and their relation with the theory of contributing divisors of Tucker [25]. The main result of this section is the fact that, among all the contributing divisors associated to a jumping number that give the same ideal, there is a minimal one. For example, critical divisors are of this type. It turns out that these minimal contributing divisors are all contained in the minimal jumping divisor and inherit the same invariance property with respect to log-resolutions of the ideal.

\section{Preliminaries}

Let $X$ be a normal complex surface, and $O$ a point where $X$ has at worst a rational singularity. That is, there exists a desingularization $\pi: X^{\prime} \rightarrow X$ such that the stalk at $O$ of the higher direct image $R^{1} \pi_{*} \mathcal{O}_{X^{\prime}}$ is zero. This property is then satisfied for any desingularization. The theory of rational singularities was introduced by Artin [4] and further developed by Lipman [19]. Another reference that we will follow closely is [22].

Let $\mathfrak{a} \subseteq \mathcal{O}_{X}$ be an ideal sheaf. Denote by $\mathfrak{m}=\mathfrak{m}_{X, O} \subseteq \mathcal{O}_{X, O}$ the maximal ideal of the local ring $\mathcal{O}_{X, O}$ at $O$. Throughout this work we will often consider the case where $\mathfrak{a} \subseteq \mathfrak{m}$ is an $\mathfrak{m}$-primary ideal, which can be identified with an ideal sheaf that equals $\mathcal{O}_{X}$ outside the point $O$ (we will use both languages interchangeably, depending on the context). Recall that a log-resolution of the pair $(X, \mathfrak{a})$ (or of $\mathfrak{a}$ for short) is a proper birational morphism $\pi: X^{\prime} \rightarrow X$ such that

(i) $X^{\prime}$ is smooth,

(ii) the preimage of $\mathfrak{a}$ is locally principal, that is, $\mathfrak{a} \cdot \mathcal{O}_{X^{\prime}}=\mathcal{O}_{X^{\prime}}(-F)$ for some effective Cartier divisor $F$, and

(iii) $F+E$ is a divisor with simple normal crossings support where $E=\operatorname{Exc}(\pi)$ is the exceptional locus.

From now on, consider a given log-resolution of $\mathfrak{a}$. Since the point $O$ has (at worst) a rational singularity, the exceptional locus $E$ is a tree of smooth rational 
curves $E_{1}, \ldots, E_{r}$. Furthermore, the matrix of intersections $\left(E_{i} \cdot E_{j}\right)_{1 \leq i, j \leq r}$ is negative-definite.

Let $\operatorname{Div}\left(X^{\prime}\right)$ be the group of integral divisors in $X^{\prime}$, that is, divisors of the form $D=\sum_{i} d_{i} E_{i}$ where the $E_{i}$ are pairwise different (not necessarily exceptional) prime divisors and $d_{i} \in \mathbb{Z}$. Among them, we will consider divisors in the lattice $\Lambda:=\mathbb{Z} E_{1} \oplus \cdots \oplus \mathbb{Z} E_{r}$ of exceptional divisors, and we will simply refer them as divisors with exceptional support. Any divisor $D \in \operatorname{Div}\left(X^{\prime}\right)$ has a decomposition $D=D_{\text {exc }}+D_{\text {aff }}$ into its exceptional and affine part $^{2}$ according to its support. Our main example is the divisor $F$ such that $\mathfrak{a} \cdot \mathcal{O}_{X^{\prime}}=\mathcal{O}_{X^{\prime}}(-F)$. In this case we will denote its exceptional and affine part as

$$
F_{\text {exc }}=\sum_{i=1}^{r} e_{i} E_{i} \quad \text { and } \quad F_{\text {aff }}=\sum_{i=r+1}^{s} e_{i} E_{i},
$$

where, by definition, $e_{i}$ are nonnegative integers. Whenever $\mathfrak{a}$ is an $\mathfrak{m}$-primary ideal, the divisor $F$ is just supported on the exceptional locus, that is, $F=F_{\text {exc }}$.

REMARK 2.1. Let $C: f=0$ be a curve defined by an element $f \in \mathcal{O}_{X, O}$. The total transform of $C$ is the pull-back $\bar{C}:=\pi^{*} C$, and its strict transform $C^{\prime}$ is the closure of $\pi^{-1}(C-\{O\})$. The total transform has a presentation $\bar{C}=C^{\prime}+\bar{C}_{\mathrm{exc}}=$ $C^{\prime}+\sum d_{i} E_{i}$ where the weights $v_{i}(f):=d_{i}$ are the values of the curve $C$ at $E_{i}$. Recall that $f \in \mathfrak{a}$ whenever $C^{\prime}+\bar{C}_{\mathrm{exc}} \geq F$ and $f$ is generic in $\mathfrak{a}$ if $\bar{C}_{\mathrm{exc}}=F_{\mathrm{exc}}$ and $C^{\prime}-F_{\text {aff }}$ has no singular points.

More generally, we will also consider $\mathbb{Q}$-divisors in $\operatorname{Div}_{\mathbb{Q}}\left(X^{\prime}\right)=\operatorname{Div}\left(X^{\prime}\right) \otimes_{\mathbb{Z}} \mathbb{Q}$ or divisors in the $\mathbb{Q}$-vector space $\Lambda_{\mathbb{Q}}:=\mathbb{Q} E_{1} \oplus \cdots \oplus \mathbb{Q} E_{r}$. The main example will be the relative canonical divisor $K_{\pi}$. Indeed, the definition of $K_{\pi}$ is quite subtle if $O$ is singular because at first sight we can only define a canonical divisor $K_{X}$ of $X$ as a Weil divisor. Since rational singularities are in particular $\mathbb{Q}$-factorial, there exists a positive integer $m$ such that $m K_{X}$ is Cartier, which can be pulled back to $X^{\prime}$ and allows us to define $K_{\pi}=K_{X^{\prime}}-\frac{1}{m} \pi^{*}\left(m K_{X}\right)$. Alternatively,

$$
K_{\pi}=\sum_{i=1}^{r} k_{i} E_{i}
$$

is supported on the exceptional locus $E$ and must satisfy

$$
\left(K_{\pi}+E_{i}\right) \cdot E_{i}=\left(\sum_{j=1}^{r} k_{j} E_{j} \cdot E_{i}\right)+E_{i}^{2}=-2
$$

for every exceptional component $E_{i}$ because of the adjunction formula. This property indeed characterizes $K_{\pi}$ because the intersection form on $E$ is negativedefinite, and therefore the system defined by equations (2.1) has a unique solution $\left(k_{1}, \ldots, k_{r}\right)$. However, the $k_{i}$ are not necessarily integral and can even be negative. In the case that $k_{i}>-1$ (resp. $k_{i} \geq-1$ ) for all $E_{i}$, we say that $X$ has a log-terminal singularity (resp. log-canonical singularity) at $O$.

\footnotetext{
${ }^{2}$ We follow the terminology of Lipman-Watanabe [20]
} 
For any $\mathbb{Q}$-divisor $D=\sum_{i} d_{i} E_{i} \in \operatorname{Div}_{\mathbb{Q}}\left(X^{\prime}\right)$, we denote its round-down and round-up as

$$
\lfloor D\rfloor=\sum_{i}\left\lfloor d_{i}\right\rfloor E_{i} \quad \text { and } \quad\lceil D\rceil=\sum_{i}\left\lceil d_{i}\right\rceil E_{i} .
$$

The fractional part of $D$ is then $\{D\}=D-\lfloor D\rfloor=\sum_{i}\left\{d_{i}\right\} E_{i}$. In the sequel we will denote the value of each component $E_{i}$ of $D$ as $v_{E_{i}}(D):=d_{i}$. If no confusion arises, we will simply denote the value of the components as $v_{i}(D):=v_{E_{i}}(D)$.

\subsection{Dual Graph}

The combinatorics of the log-resolution of $\mathfrak{a}$ can be encoded using the so-called dual graph. This is a rooted tree where the vertices represent the irreducible components $E_{i} \leq F$ and two vertices are joined by an edge if the corresponding divisors intersect.

Given any component $E_{i}$, we will denote by $\operatorname{Adj}\left(E_{i}\right)$ the set of components $E_{j}, j \neq i$, sharing an edge with $E_{i}$, that is $E_{i} \cdot E_{j}=1$, and by

$$
a\left(E_{i}\right)=\# \operatorname{Adj}\left(E_{i}\right)=E_{i} \cdot\left(F^{\mathrm{red}}-E_{i}\right)
$$

the number of such components, which is the valence of the vertex representing $E_{i}$, where $F^{\text {red }}$ denotes de reduced divisor with the same support as $F$. An end of the dual graph is nothing but a vertex with valence 1 , that is, a vertex $E_{i}$ such that $a\left(E_{i}\right)=1$. More generally, for any effective subdivisor $D=E_{i_{1}}+\cdots+E_{i_{m}} \leq F$, we define

$$
\operatorname{Adj}_{D}\left(E_{i}\right)=\left\{E_{j} \leq D \mid E_{i} \cdot E_{j}=1\right\}
$$

and $a_{D}\left(E_{i}\right)=\# \operatorname{Adj}_{D}\left(E_{i}\right)$. We denote by $v_{D}=m$ (resp. $\left.a_{D}\right)$ the number of components of $D$ (resp. the number of intersections between two components of $D$ ). Since the dual graph is a tree, it is clear that

$$
\sum_{E_{i} \leq D} a_{D}\left(E_{i}\right)=2 a_{D}
$$

and that $v_{D}-a_{D}$ equals the number of connected components of $D$. An end of the subgraph associated to $D$ is a vertex with valence 1 or 0 , the later meaning that $E_{i}$ is an isolated component of $D$.

For any exceptional component $E_{i}$, we define the excess (of $\mathfrak{a}$ ) at $E_{i}$ as $\rho_{i}=$ $-F \cdot E_{i}$. It can be interpreted as the number of branches of the strict transform of a curve defined by a generic element $f \in \mathfrak{a}$ that intersects the component $E_{i}$. Indeed, if its total transform is $\bar{C}=C^{\prime}+F$, then $0=\bar{C} \cdot E_{i}=C^{\prime} \cdot E_{i}+F \cdot E_{i}=$ $C^{\prime} \cdot E_{i}-\rho_{i}$, which proves the claim.

There are two kinds of exceptional divisors that will play a special role:

- A component $E_{i}$ of $E$ is a rupture component if $a\left(E_{i}\right) \geq 3$, that is, it intersects at least three more components of $E$ (different from $E_{i}$ ).

- We say that $E_{i}$ is dicritical if $\rho_{i}>0$. By [19] dicritical components correspond to Rees valuations.

We also mention that nonexceptional components also correspond to Rees valuations. 


\subsection{Complete Ideals and Antinef Divisors}

Given an effective $\mathbb{Q}$-divisor $D=\sum d_{i} E_{i} \in \operatorname{Div}_{\mathbb{Q}}\left(X^{\prime}\right)$, we may consider its associated (sheaf) ideal $\pi_{*} \mathcal{O}_{X^{\prime}}(-D):=\pi_{*} \mathcal{O}_{X^{\prime}}(-\lceil D\rceil)$. Its stalk at $O$ is

$$
I_{D}:=\left\{f \in \mathcal{O}_{X, O} \mid v_{i}(f) \geq\left\lceil d_{i}\right\rceil \text { for all } E_{i} \leq D\right\} .
$$

This is a complete ideal of $\mathcal{O}_{X, O}$ that is m-primary whenever $D$ has exceptional support, that is, $D \in \Lambda_{\mathbb{Q}}$. Any two divisors $D, D^{\prime} \in \operatorname{Div}_{\mathbb{Q}}\left(X^{\prime}\right)$ defining the same complete ideal $\pi_{*} \mathcal{O}_{X^{\prime}}(-D)=\pi_{*} \mathcal{O}_{X^{\prime}}\left(-D^{\prime}\right)$ are called equivalent divisors.

In the equivalence class of a given divisor, we may find a unique maximal representative. First, recall that an effective divisor with integral coefficients $D \in$ $\operatorname{Div}\left(X^{\prime}\right)$ is called antinef if $-D \cdot E_{i} \geq 0$ for every exceptional prime divisor $E_{i}$. It is worth pointing out that the affine part of $D=D_{\text {exc }}+D_{\text {aff }}$ satisfies $D_{\text {aff }} \cdot E_{i} \geq 0$. Therefore, $D$ is antinef whenever $-D_{\text {exc }} \cdot E_{i} \geq D_{\text {aff }} \cdot E_{i}$.

In the work of Lipman (see $[19, \S 18]$ ) we may find the following correspondence that we will heavily use throughout this work.

THEOREM 2.2. There is a one-to-one correspondence between antinef divisors in $\operatorname{Div}\left(X^{\prime}\right)$ and complete ideals in $\mathcal{O}_{X, O}$. In particular, antinef divisors in $\Lambda$ correspond to $\mathfrak{m}$-primary complete ideals.

In order to find the representative in the equivalence class of a given divisor $D \in$ $\operatorname{Div}_{\mathbb{Q}}\left(X^{\prime}\right)$, we will consider its so-called antinef closure $\widetilde{D}$. The existence of such a divisor is a consequence of the following results that can be found in $[19, \S 18]$, but we also refer to [24] and [20] for more insight.

Lemma 2.3. For any effective $\mathbb{Q}$-divisor $D \in \operatorname{Div}_{\mathbb{Q}}\left(X^{\prime}\right)$, there exists a unique minimal integral antinef divisor $\widetilde{D} \in \operatorname{Div}\left(X^{\prime}\right)$ satisfying $\widetilde{D} \geq D$ that is called the antinef closure of $D$. In particular, any antinef divisor $D^{\prime}$ such that $D^{\prime} \geq D$ must satisfy $D^{\prime} \geq \widetilde{D} \geq D$.

Proposition 2.4. An effective $\mathbb{Q}$-divisor $D \in \operatorname{Div}_{\mathbb{Q}}\left(X^{\prime}\right)$ and its antinef closure $\widetilde{D} \in \operatorname{Div}\left(X^{\prime}\right)$ are equivalent, that is,

$$
\pi_{*} \mathcal{O}_{X^{\prime}}(-D)=\pi_{*} \mathcal{O}_{X^{\prime}}(-\widetilde{D}) .
$$

One of the advantages of working with antinef divisors is that they provide the following characterization for the inclusion (or strict inclusion) of two given complete ideals.

Proposition 2.5. Let $D_{1}, D_{2}$ be two antinef divisors in $\operatorname{Div}\left(X^{\prime}\right)$. Then:

(i) $\pi_{*} \mathcal{O}_{X^{\prime}}\left(-D_{1}\right) \supseteq \pi_{*} \mathcal{O}_{X^{\prime}}\left(-D_{2}\right)$ if and only if $D_{1} \leq D_{2}$.

(ii) $\pi_{*} \mathcal{O}_{X^{\prime}}\left(-D_{1}\right) \nsupseteq \pi_{*} \mathcal{O}_{X^{\prime}}\left(-D_{2}\right)$ if and only if $D_{1}<D_{2}$.

For nonantinef divisors, we can only claim the following implication:

Proposition 2.6. Let $D_{1}, D_{2}$ be two divisors in $\operatorname{Div}_{\mathbb{Q}}\left(X^{\prime}\right)$ such that $D_{1} \leq D_{2}$. Then: 
(i) $\pi_{*} \mathcal{O}_{X^{\prime}}\left(-D_{1}\right) \supseteq \pi_{*} \mathcal{O}_{X^{\prime}}\left(-D_{2}\right)$.

(ii) $\widetilde{D_{1}} \leq \widetilde{D_{2}}$.

The converses to these results are no longer true.

In general, the divisors that will be considered in this work are not antinef. In order to compute their antinef closure, we will use an inductive procedure called unloading that was already described in the work of Enriques [7, IV.II.17] (for the case of smooth varieties) and Laufer's procedure to compute the fundamental cycle [17] (for varieties with rational singularities). Here we will present a new version, which is a generalization of both the unloading procedures described by Casas-Alvero [5, §4.6] (for smooth varieties) and Reguera [22] (for varieties with rational singularities).

Unloading Procedure. Let $D \in \operatorname{Div}_{\mathbb{Q}}\left(X^{\prime}\right)$ be any $\mathbb{Q}$-divisor. Its excess at the exceptional prime divisor $E_{i}$ is the integer $\rho_{i}=-\lceil D\rceil \cdot E_{i}$. Denote by $\Theta$ the set of exceptional components $E_{i} \leq D$ with negative excesses, that is,

$$
\Theta:=\left\{E_{i} \leq D_{\text {exc }} \mid \rho_{i}=-\lceil D\rceil \cdot E_{i}<0\right\} .
$$

To unload values on this set is to consider the new divisor

$$
D^{\prime}=\lceil D\rceil+\sum_{E_{i} \in \Theta} n_{i} E_{i},
$$

where $n_{i}=\left\lceil\rho_{i} / E_{i}^{2}\right\rceil$. Notice that $n_{i}$ is the least integer number such that

$$
\left(\lceil D\rceil+n_{i} E_{i}\right) \cdot E_{i}=-\rho_{i}+n_{i} E_{i}^{2} \leq 0 .
$$

REMARK 2.7. Casas-Alvero considered at each step just one component with negative excess. Reguera also considered one component with negative excess, but in her case she also imposed $n_{i}=1$ at each step. In this sense, our approach is more economic from a computational point of view. Furthermore, our procedure allows unloading on divisors with affine part, ${ }^{3}$ which will enable us to treat in a unified way multiplier ideals of both curves and not necessarily $\mathfrak{m}$-primary complete ideals.

The correctness of the unloading procedure is a consequence of the following results.

Proposition 2.8. Let $D^{\prime}$ be the divisor obtained from a divisor $D \in \operatorname{Div}_{\mathbb{Q}}\left(X^{\prime}\right)$ after one single unloading step. Then $I_{D^{\prime}}=I_{D}$.

Proof. It is clear from its construction that $I_{D^{\prime}} \subseteq I_{D}$. Pick $f \in I_{D}$ and let $\bar{C}=$ $C^{\prime}+\bar{C}_{\text {exc }}$ be the total transform of the curve $C$ defined by $f=0$. We have $v_{i}(f) \geq$ $v_{i}(\lceil D\rceil) \geq v_{i}(D)$ for all $E_{i}$. Consider any exceptional divisor $E_{i}$ where $D$ has negative excess. From the inequality $\left(\bar{C}_{\mathrm{exc}}-v_{i}(f) E_{i}\right) \cdot E_{i} \geq\left(\lceil D\rceil-v_{i}(\lceil D\rceil) E_{i}\right)$. $E_{i}$ we deduce

$$
-v_{i}(f) E_{i} \cdot E_{i} \geq\left(\lceil D\rceil-v_{i}(\lceil D\rceil) E_{i}\right) \cdot E_{i}
$$

\footnotetext{
${ }^{3}$ Our method also differs from that considered by Lipman and Watanabe [20].
} 
just because $\bar{C}_{\text {exc }} \cdot E_{i} \leq 0$. Equivalently, $\left(\lceil D\rceil+\left(v_{i}(f)-v_{i}(\lceil D\rceil)\right) E_{i}\right) \cdot E_{i} \leq 0$, so it follows that $n_{i} \leq v_{i}(f)-v_{i}(\lceil D\rceil)$. In particular, $n_{i}+v_{i}(\lceil D\rceil) \leq v_{i}(f)$ and $f \in I_{D^{\prime}}$.

Proposition 2.9. The antinef closure $\widetilde{D}$ of a divisor $D \in \operatorname{Div}_{\mathbb{Q}}\left(X^{\prime}\right)$ is achieved after finitely many unloading steps.

Proof. We want to show that the divisors in the sequence

$$
D \leq D_{1}=\lceil D\rceil<\cdots<D_{t}<D_{t+1}<\cdots
$$

obtained during the unloading procedure are all contained in the antinef closure $\widetilde{D}$. Then the result will follow since both $D_{1}$ and $\widetilde{D}$ have integral coefficients and the inequalities in the unloading sequence are strict. Clearly, $D_{1} \leq \widetilde{D}$, and suppose that $D_{t} \leq \widetilde{D}$. Notice that for any component $E_{i} \leq D_{t}$ with negative excess, we have $\left(\widetilde{D}-D_{t}\right) \cdot E_{i} \leq-D_{t} \cdot E_{i}$. Then, if we denote $\widetilde{D}-D_{t}=\sum_{i} m_{i} E_{i}$, then the previous inequality becomes

$$
\begin{aligned}
\left(\widetilde{D}-D_{t}\right) \cdot E_{i} & =\left(m_{i} E_{i}+\sum_{j \neq i} m_{j} E_{j}\right) \cdot E_{i} \\
& =m_{i} E_{i}^{2}+\sum_{j \neq i} m_{j} E_{j} \cdot E_{i} \leq-D_{t} \cdot E_{i} .
\end{aligned}
$$

Then, using that $\sum_{j \neq i} m_{j} E_{j} \cdot E_{i} \geq 0$, we get

$$
m_{i} \geq\left\lceil\frac{-D_{t} \cdot E_{i}}{E_{i}^{2}}\right\rceil,
$$

where we used the fact that $D_{t}$ and $\widetilde{D}$ have integer coefficients. It follows that $D_{t+1}$ is also contained in $\widetilde{D}$.

\subsection{Multiplier Ideals}

Let $\pi: X^{\prime} \rightarrow X$ be a log-resolution of an ideal $\mathfrak{a} \subseteq \mathcal{O}_{X}$, and let $F$ be the divisor such that $\mathfrak{a} \cdot \mathcal{O}_{X^{\prime}}=\mathcal{O}_{X^{\prime}}(-F)$. The multiplier ideal (sheaf) associated to $\mathfrak{a}$ and some rational number $\lambda \in \mathbb{Q}_{>0}$ is defined as ${ }^{4}$

$$
\mathcal{J}\left(\mathfrak{a}^{\lambda}\right)=\pi_{*} \mathcal{O}_{X^{\prime}}\left(\left\lceil K_{\pi}-\lambda F\right\rceil\right) .
$$

For a detailed overview of the theory of multiplier ideals and the properties they satisfy, we must refer to the book of Lazarsfeld [18]. For more details in the case that $X$ has rational singularities, we also recommend to take a look at [24] and [25].

The definition of multiplier ideals is independent of the choice of log resolution. For simplicity, we will always fix a given resolution. Multiplier ideals are complete, and they are invariants up to integral closure, that is, $\mathcal{J}\left(\mathfrak{a}^{\lambda}\right)=\mathcal{J}\left(\overline{\mathfrak{a}}^{\lambda}\right)$; therefore, without loss of generality, we may always assume that the ideal $\mathfrak{a}$ is

\footnotetext{
${ }^{4}$ By an abuse of notation, we will also denote $\mathcal{J}\left(\mathfrak{a}^{\lambda}\right)$ its stalk at $O$, so we will omit the word "sheaf"
} if no confusion arises. 
complete. Moreover, if $\mathfrak{a}$ is $\mathfrak{m}$-primary, then it follows that its associated multiplier ideals $\mathcal{J}\left(\mathfrak{a}^{\lambda}\right)$ are $\mathfrak{m}$-primary as well.

Some other important properties of multiplier ideals that we will use in this work are:

- Local vanishing theorem: $R^{i} \pi_{*} \mathcal{O}_{X^{\prime}}\left(\left\lceil K_{\pi}-\lambda F\right\rceil\right)=0$ for all $i>0$ and all $\lambda \in$ $\mathbb{Q}>0$.

- Skoda's theorem: $\mathcal{J}\left(\mathfrak{a}^{\lambda}\right)=\mathfrak{a} \cdot \mathcal{J}\left(\mathfrak{a}^{\lambda-1}\right)$ for all $\lambda>\operatorname{dim} \mathcal{O}_{X, O}=2$.

For the case of principal ideals, there is another version of Skoda's theorem, which states that $\mathcal{J}\left(\mathfrak{a}^{\lambda}\right)=\mathfrak{a} \cdot \mathcal{J}\left(\mathfrak{a}^{\lambda-1}\right)$ for all $\lambda \geq 1$. In particular, we have periodicity of jumping numbers.

Multiplier ideals come with an attached set of invariants that were studied systematically by Ein, Lazarsfeld, Smith, and Varolin [6]. Clearly,

$$
\left\lceil K_{\pi}-\lambda F\right\rceil \geq\left\lceil K_{\pi}-(\lambda+\varepsilon) F\right\rceil
$$

for any $\varepsilon>0$, with equality if $\varepsilon$ is small enough. Therefore, the multiplier ideals form a discrete nested sequence of ideals

$$
\mathcal{O}_{X, O} \supseteq \mathcal{J}\left(\mathfrak{a}^{\lambda_{0}}\right) \supseteq \mathcal{J}\left(\mathfrak{a}^{\lambda_{1}}\right) \supseteq \mathcal{J}\left(\mathfrak{a}^{\lambda_{2}}\right) \supseteq \cdots \supseteq \mathcal{J}\left(\mathfrak{a}^{\lambda_{i}}\right) \nsupseteq \cdots
$$

indexed by an increasing sequence of rational numbers $0=\lambda_{0}<\lambda_{1}<\lambda_{2}<\cdots$ such that, for any $c \in\left[\lambda_{i}, \lambda_{i+1}\right)$,

$$
\mathcal{J}\left(\mathfrak{a}^{\lambda_{i}}\right)=\mathcal{J}\left(\mathfrak{a}^{c}\right) \supsetneq \mathcal{J}\left(\mathfrak{a}^{\lambda_{i+1}}\right) .
$$

The $\lambda_{i}$ are the so-called jumping numbers of the ideal $\mathfrak{a}$, and the first jumping number $\lambda_{1}=\operatorname{lct}(\mathfrak{a})$ is the log-canonical threshold of $\mathfrak{a}$.

\subsection{Contributing Divisors}

The jumps between multiplier ideals necessarily must occur at rational numbers $\lambda \in \mathbb{Q}$, which causes the strict inclusion of divisors

$$
\left\lceil K_{\pi}-\lambda F\right\rceil<\left\lceil K_{\pi}-(\lambda-\varepsilon) F\right\rceil
$$

for any $\varepsilon$. If we take a close look at $F=F_{\text {exc }}+F_{\text {aff }}$, then these rational numbers must belong to the set of candidate jumping numbers

$$
\left\{\frac{k_{i}+m}{e_{i}} \mid m \in \mathbb{Z}_{>0}\right\} .
$$

Notice that for nonexceptional components $E_{i} \leq F_{\text {aff }}$, we have $k_{i}=0$, and their corresponding candidates $\left\{m / e_{i} \mid m \in \mathbb{Z}_{>0}\right\}$ are indeed jumping numbers.

It is easy to check that not every candidate jumping number (coming from the exceptional part) is necessarily a jumping number. To separate the wheat from the chaff, Tucker [25] developed the notion of divisor that contributes to a jumping number, building upon previous work by Smith and Thompson [23].

DEFINITION 2.10. A positive rational number $\lambda$ is a candidate jumping number for a reduced divisor $G \leq F$ if it satisfies $\lambda e_{i}-k_{i} \in \mathbb{Z}_{>0}$ for any component $E_{i} \leq G$. 
Definition 2.11 [25, Def. 3.1]. A reduced divisor $G \leq F$ for which $\lambda$ is a candidate jumping number is said to contribute to $\lambda$ if

$$
\pi_{*} \mathcal{O}_{X^{\prime}}\left(\left\lceil K_{\pi}-\lambda F\right\rceil+G\right) \nsupseteq \mathcal{J}\left(\mathfrak{a}^{\lambda}\right) .
$$

Moreover, this contribution is critical if for any divisor $0 \leq G^{\prime}<G$, we have

$$
\pi_{*} \mathcal{O}_{X^{\prime}}\left(\left\lceil K_{\pi}-\lambda F\right\rceil+G^{\prime}\right)=\mathcal{J}\left(\mathfrak{a}^{\lambda}\right) .
$$

Most often, we will simply say that $G$ is just a contributing or a critical divisor associated to $\lambda$. Critical divisors define complete ideals very close to a multiplier ideal in a precise sense that will be explained in the forthcoming Corollary 5.5 in Section 5. We may identify critical divisors with exceptional support through the following numerical characterization.

Proposition 2.12 [25, Thm. 4.3]. Let $\lambda$ be a candidate jumping number for $a$ reduced divisor $G \in \Lambda$ with connected support.

- If $G=E_{i}$ is prime, then $E_{i}$ is a critical divisor for $\lambda$ if and only if

$$
\left(\left\lceil K_{\pi}-\lambda F\right\rceil+E_{i}\right) \cdot E_{i} \geq 0 .
$$

- If $G$ is reducible, then $G$ is a critical divisor for $\lambda$ if and only if

$$
\left(\left\lceil K_{\pi}-\lambda F\right\rceil+G\right) \cdot E_{i}=0
$$

for all divisors $E_{i}$ in the support of $G$.

Moreover, critical divisors with exceptional support satisfy a nice geometric property when viewed in the dual graph.

Proposition 2.13 [25, Cor. 4.2 and Thm. 5.1]. Let $G$ be a critical divisor for a jumping number $\lambda$. Then $G$ is a connected chain in the dual graph of the logresolution of $\mathfrak{a}$ whose ends must be either rupture or dicritical divisors.

Using all these properties, Tucker provides a simple algorithm to compute the set of all jumping numbers (see $[25, \S 6])$. It boils down to the following steps:

Algorithm 2.14 (jumping numbers).

Input: A log-resolution of an ideal $\mathfrak{a} \subseteq \mathcal{O}_{X, O}$.

Output: List of jumping numbers of $\mathfrak{a}$.

- Jumping number:

- Compute the candidate jumping numbers for $F_{\text {exc }}$.

- Find all possible critical divisors using Proposition 2.13.

- Find which candidate jumping numbers can be realized as a jumping number associated to these critical divisors using Proposition 2.12.

- Plug in those jumping numbers coming from $F_{\text {aff }}$. 


\section{An Algorithm to Compute Jumping Numbers and Multiplier Ideals}

The aim of this section is to compute the jumping numbers and their corresponding multiplier ideals of any given ideal $\mathfrak{a} \subseteq \mathcal{O}_{X, O}$. For such a purpose, we fix a $\log$-resolution $\pi: X^{\prime} \rightarrow X$ of our ideal $\mathfrak{a}$. The main ingredients we will have to deal with are the relative canonical divisor $K_{\pi}=\sum_{i=1}^{r} k_{i} E_{i} \in \Lambda_{\mathbb{Q}}$ and the divisor $F \in \operatorname{Div}\left(X^{\prime}\right)$ such that $\mathfrak{a} \mathcal{O}_{X^{\prime}}=\mathcal{O}_{X^{\prime}}(-F)$. Recall that we have a decomposition

$$
F=F_{\text {exc }}+F_{\text {aff }}=\sum_{i=1}^{r} e_{i} E_{i}+\sum_{i=r+1}^{s} e_{i} E_{i}
$$

in terms of its exceptional and affine support.

We will provide a very simple algorithm that allows us to construct sequentially the chain of multiplier ideals ${ }^{5}$

$$
\mathcal{O}_{X, O} \supseteq \mathcal{J}\left(\mathfrak{a}^{\lambda_{0}}\right) \supseteq \mathcal{J}\left(\mathfrak{a}^{\lambda_{1}}\right) \supseteq \mathcal{J}\left(\mathfrak{a}^{\lambda_{2}}\right) \supseteq \cdots \supseteq \mathcal{J}\left(\mathfrak{a}^{\lambda_{i}}\right) \supseteq \cdots
$$

When $X$ is a smooth surface, or even when $X$ has a log-terminal singularity at $O$, the multiplier ideal associated to $\lambda_{0}=0$ is the whole ring, that is, $\mathcal{O}_{X, O}=\mathcal{J}\left(\mathfrak{a}^{\lambda_{0}}\right)$. In general, when $X$ has a rational singularity, we may have an strict inclusion $\mathcal{O}_{X, O} \supsetneq \mathcal{J}\left(\mathfrak{a}^{\lambda_{0}}\right)$. The starting point of our method will be describing this ideal by means of the antinef closure $D_{\lambda_{0}}=\sum e_{i}^{\lambda_{0}} E_{i}$ of $\left\lfloor-K_{\pi}\right\rfloor$, which we compute using the unloading procedure described in Section 2.2.

As a consequence of our main result (see Theorem 3.5), the log-canonical threshold satisfies the formula 6

$$
\lambda_{1}=\operatorname{lct}(\mathfrak{a})=\min _{i}\left\{\frac{k_{i}+1+e_{i}^{\lambda_{0}}}{e_{i}}\right\} .
$$

Then we describe its associated multiplier ideal $\mathcal{J}\left(\mathfrak{a}^{\lambda_{1}}\right)$ just computing the antinef closure $D_{\lambda_{1}}$ of $\left\lfloor\lambda_{1} F-K_{\pi}\right\rfloor$ using the unloading procedure. Once we have the divisor $D_{\lambda_{1}}$, we use an extension of Formula (3.1) given by Theorem 3.5, which computes the next jumping number $\lambda_{2}$. Then we only have to follow the same strategy: the antinef closure $D_{\lambda_{2}}$ of $\left\lfloor\lambda_{2} F-K_{\pi}\right\rfloor$, that is, the multiplier ideal $\mathcal{J}\left(\mathfrak{a}^{\lambda_{2}}\right)$, will allow us to compute $\lambda_{3}$ and so on.

The main idea behind our method is a simple comparison between complete ideals. Whenever we have two antinef divisors, it is easy to check whether their corresponding complete ideals satisfy a strict inclusion (see Proposition 2.5). Comparison of the ideals associated to an antinef and a nonantinef divisor is more subtle, and this is the situation that we will have to deal with in this section.

To address this problem, we will need some preliminary technical results.

${ }^{5}$ In fact, we can compute the chain inside any desired fixed range $\left[c, c^{\prime}\right] \subseteq \mathbb{R}$ :

$$
\mathcal{J}\left(\mathfrak{a}^{c}\right)=\mathcal{J}\left(\mathfrak{a}^{\lambda_{0}}\right) \nsupseteq \mathcal{J}\left(\mathfrak{a}^{\lambda_{1}}\right) \nsupseteq \cdots \supseteq \mathcal{J}\left(\mathfrak{a}^{\lambda_{r}}\right)=\mathcal{J}\left(\mathfrak{a}^{c^{\prime}}\right) .
$$

\footnotetext{
${ }^{6}$ When $X$ is smooth, or even when it has log-terminal singularities, we have $D_{\lambda_{0}}=0$, so we recover
} the well-known formula for the log-canonical threshold. 
Lemma 3.1. Let $D_{1}, D_{2}$ be two divisors in $\operatorname{Div}\left(X^{\prime}\right)$ such that $D_{1} \leq D_{2}$. Then, they have the same antinef closure $\widetilde{D_{1}}=\widetilde{D_{2}}$ if and only if $\widetilde{D_{1}} \geq D_{2}$.

Proof. Recall that, by Proposition 2.6, we already have $\widetilde{D_{1}} \leq \widetilde{D_{2}}$ just because $D_{1} \leq D_{2}$.

Assume that $\widetilde{D_{1}} \geq D_{2}$; then, by the definition of antinef closure (see Lemma 2.3) we also have $\widetilde{D_{1}} \geq \widetilde{D_{2}} \geq D_{2}$, and thus $\widetilde{D_{1}}=\widetilde{D_{2}}$. On the other hand, assume that $\widetilde{D_{1}}=\widetilde{D_{2}}$. Then, since the antinef closure of a divisor always contains it, we have $\widetilde{D_{1}}=\widetilde{D_{2}} \geq D_{2}$, as desired.

Corollary 3.2. Let $D_{1}, D_{2}$ be two divisors in $\operatorname{Div}\left(X^{\prime}\right)$ such that $D_{1} \leq D_{2}$. Then, $\widetilde{D_{1}}<\widetilde{D_{2}}$ if and only if $v_{i}\left(\widetilde{D_{1}}\right)<v_{i}\left(D_{2}\right)$ for some $E_{i}$.

Proof. Since $D_{1} \leq D_{2}$, the inclusion $\widetilde{D_{1}} \leq \widetilde{D_{2}}$ also holds. The result then follows from Lemma 3.1.

Translated into the language of complete ideals, these results give a characterization of the jump between two nested ideals, which will be a key ingredient in the proof of our results.

Proposition 3.3. Let $D_{1}, D_{2}$ be two divisors in $\operatorname{Div}\left(X^{\prime}\right)$ such that $D_{1} \leq D_{2}$. Then:

(i) $\pi_{*} \mathcal{O}_{X^{\prime}}\left(-D_{1}\right)=\pi_{*} \mathcal{O}_{X^{\prime}}\left(-D_{2}\right)$ if and only if $\widetilde{D_{1}} \geq D_{2}$.

(ii) $\pi_{*} \mathcal{O}_{X^{\prime}}\left(-D_{1}\right) \supseteq \pi_{*} \mathcal{O}_{X^{\prime}}\left(-D_{2}\right)$ if and only if $v_{i}\left(\widetilde{D_{1}}\right)<v_{i}\left(D_{2}\right)$ for some $E_{i}$.

For convenience, we also present this result in the form we will most commonly use.

COROLlary 3.4. Let $\lambda^{\prime}<\lambda$ be rational numbers. Let $D_{\lambda^{\prime}}=\sum e_{i}^{\lambda^{\prime}} E_{i}$ be the antinef closure of $\left\lfloor\lambda^{\prime} F-K_{\pi}\right\rfloor$. Then:

(i) $\mathcal{J}\left(\mathfrak{a}^{\lambda^{\prime}}\right)=\mathcal{J}\left(\mathfrak{a}^{\lambda}\right)$ if and only if $\left\lfloor\lambda e_{i}-k_{i}\right\rfloor \leq e_{i}^{\lambda^{\prime}}$ for all $E_{i}$.

(ii) $\mathcal{J}\left(\mathfrak{a}^{\lambda^{\prime}}\right) \nsupseteq \mathcal{J}\left(\mathfrak{a}^{\lambda}\right)$ if and only if $\left\lfloor\lambda e_{i}-k_{i}\right\rfloor>e_{i}^{\lambda^{\prime}}$ for some $E_{i}$.

With the technical tools stated, we are ready for the main result of this section.

TheOREM 3.5. Let $\mathfrak{a} \subseteq \mathcal{O}_{X, O}$ be an ideal, and let $D_{\lambda^{\prime}}=\sum e_{i}^{\lambda^{\prime}} E_{i}$ be the antinef closure of $\left\lfloor\lambda^{\prime} F-K_{\pi}\right\rfloor$ for a given $\lambda^{\prime} \in \mathbb{Q}_{>0}$. Then,

$$
\lambda=\min _{i}\left\{\frac{k_{i}+1+e_{i}^{\lambda^{\prime}}}{e_{i}}\right\}
$$

is the jumping number consecutive to $\lambda^{\prime}$.

Proof. Let us first check that $\lambda^{\prime}<\lambda$. Indeed, by the definition of antinef closure the integers $e_{i}^{\lambda^{\prime}}$ satisfy $\left\lfloor\lambda^{\prime} e_{i}-k_{i}\right\rfloor \leq e_{i}^{\lambda^{\prime}}$ for any $E_{i}$, and hence

$$
\lambda^{\prime}<\frac{k_{i}+1+e_{i}^{\lambda^{\prime}}}{e_{i}} \text {. }
$$


Thus, we have an inclusion of ideals $\mathcal{J}\left(\mathfrak{a}^{\lambda^{\prime}}\right) \supseteq \mathcal{J}\left(\mathfrak{a}^{\lambda}\right)$. Notice that for those divisors $E_{i}$ where the minimum is achieved, we have

$$
\left\lfloor\lambda e_{i}-k_{i}\right\rfloor=1+e_{i}^{\lambda^{\prime}}>e_{i}^{\lambda^{\prime}},
$$

so the inclusion of ideals is strict by Corollary 3.4. To conclude that $\lambda$ is the jumping number immediately after $\lambda^{\prime}$, we have to show that for any $c \in \mathbb{R}$ with $\lambda^{\prime} \leq c<\lambda$, we have $\mathcal{J}\left(\mathfrak{a}^{\lambda^{\prime}}\right)=\mathcal{J}\left(\mathfrak{a}^{c}\right)$. Suppose the contrary, that is, $\mathcal{J}\left(\mathfrak{a}^{\lambda^{\prime}}\right) \supseteq$ $\mathcal{J}\left(\mathfrak{a}^{c}\right)$. By Corollary 3.4 this $c$ should satisfy $\left\lfloor\lambda e_{i}-k_{i}\right\rfloor>e_{i}^{\lambda^{\prime}}$ or, equivalently, $c \geq$ $\left(k_{i}+1+e_{i}^{\lambda^{\prime}}\right) / e_{i}$ for some $E_{i}$, and this contradicts the fact that $\lambda$ is the minimum of these rational numbers.

This result for the case $\lambda^{\prime}=0$ gives a mild generalization of the well-known formula for the log-canonical threshold in the smooth case. We point out that the antinef closure of $\left\lfloor-K_{\pi}\right\rfloor$ is 0 whenever $X$ is smooth or, more generally, when it has log-terminal singularities.

Corollary 3.6. Let $\mathfrak{a} \subseteq \mathcal{O}_{X}, O$ be an ideal. Let $D_{\lambda_{0}}=\sum e_{i}^{\lambda_{0}} E_{i}$ be the antinef closure of $\left\lfloor-K_{\pi}\right\rfloor$. Then,

$$
\operatorname{lct}(\mathfrak{a})=\min _{i}\left\{\frac{k_{i}+1+e_{i}^{\lambda_{0}}}{e_{i}}\right\} .
$$

Another easy application of the results stated is the following result, which should be well known to experts.

Corollary 3.7. Let $\lambda_{1}$ be the log-canonical threshold of an ideal $\mathfrak{a} \subseteq \mathcal{O}_{X, O}$ and assume that $X$ has at most a log-terminal singularity at $O$. Then $\mathcal{J}\left(\mathfrak{a}^{\lambda_{1}}\right)=\mathfrak{m}$.

Proof. Since $X$ has at most a log-terminal singularity, the log-canonical threshold is

$$
\operatorname{lct}(\mathfrak{a})=\lambda_{1}=\min _{i}\left\{\frac{k_{i}+1}{e_{i}}\right\},
$$

so it satisfies $\lambda_{1} \leq\left(k_{i}+1\right) / e_{i}$ for any divisor $E_{i}$, and equality is achieved at least for a given divisor. In particular, for all $E_{i}$, we have

$$
\left\lfloor\lambda_{1} e_{i}-k_{i}\right\rfloor \leq 1 \text {. }
$$

It follows from Proposition 2.6 that $\mathfrak{m} \subseteq \mathcal{J}\left(\mathfrak{a}^{\lambda_{1}}\right) \varsubsetneqq \mathcal{O}_{X, O}$, and we get the desired result.

For non log-terminal singularities, we can find examples where the codimension as $\mathbb{C}$-vector spaces of $\mathcal{J}\left(\mathfrak{a}^{\lambda_{0}}\right) \supseteq \mathcal{J}\left(\mathfrak{a}^{\lambda_{1}}\right)$ is greater than 1 (see Example 3.10).

Combining Theorem 3.5 with the unloading procedure described in Section 2.2, we can describe a very simple algorithm that allows us to compute the chain of multiplier ideals:

Algorithm 3.8 (jumping numbers and multiplier ideals).

Input: A log-resolution of an ideal $\mathfrak{a} \subseteq \mathcal{O}_{X, O}$.

Output: List of jumping numbers of $\mathfrak{a}$ and its corresponding multiplier ideals. 
Set $\lambda_{0}=0$ and compute the antinef closure $D_{\lambda_{0}}=\sum e_{i}^{\lambda_{0}} E_{i}$ of $\left\lfloor-K_{\pi}\right\rfloor$ using the unloading procedure. From $j=1$, incrementing by 1 ,

- Stepj

- Jumping number: Compute

$$
\lambda_{j}=\min _{i}\left\{\frac{k_{i}+1+e_{i}^{\lambda_{j-1}}}{e_{i}}\right\} .
$$

- Multiplier ideal: Compute the antinef closure $D_{\lambda_{j}}=\sum e_{i}^{\lambda_{j}} E_{i}$ of $\left\lfloor\lambda_{j} F-\right.$ $\left.K_{\pi}\right\rfloor$ using the unloading procedure.

Notice that we may also find all the multiplier ideals in any given interval $\left[c^{\prime}, c\right]$ of the real line. In this case, our starting point would be computing the antinef closure $D_{c^{\prime}}$ of $\left\lfloor c^{\prime} F-K_{\pi}\right\rfloor$. To illustrate this method, we consider an easy example in a smooth variety.

EXAMPle 3.9. Consider the ideal $\mathfrak{a}=\left(x^{2} y^{2}, x^{5}, y^{5}, x y^{4}, x^{4} y\right) \subseteq \mathbb{C}\{x, y\}$. We represent the relative canonical divisor $K_{\pi}$ and the divisor $F$ in the dual graph as follows:

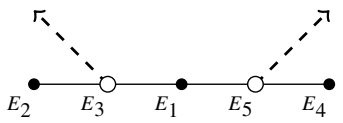

Vertex ordering

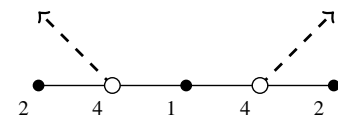

$K_{\pi}$

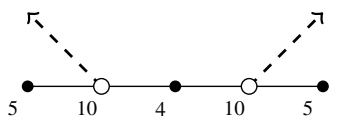

$F$

The blank dots correspond to dicritical divisors, and their excesses are represented by broken arrows. ${ }^{7}$ For simplicity, we will collect the values of any divisor in a vector. To start with, we have $K_{\pi}=(1,2,4,2,4)$ and $F=(4,5,10,5,10)$. In the algorithm we will have to perform some unloading steps, so we will have to consider the intersection matrix $M=\left(E_{i} \cdot E_{j}\right)_{1 \leq i, j \leq 5}$,

$$
M=\left(\begin{array}{ccccc}
-5 & 0 & 1 & 0 & 1 \\
0 & -2 & 1 & 0 & 0 \\
1 & 1 & -1 & 0 & 0 \\
0 & 0 & 0 & -2 & 1 \\
1 & 0 & 0 & 1 & -1
\end{array}\right)
$$

The algorithm is performed as follows:

- We start computing the log-canonical threshold:

$$
\lambda_{1}=\operatorname{lct}(\mathfrak{a})=\min _{i}\left\{\frac{k_{i}+1}{e_{i}}\right\}=\min _{i}\left\{\frac{2}{4}, \frac{3}{5}, \frac{5}{10}, \frac{3}{5}, \frac{5}{10}\right\}=\frac{1}{2} .
$$

The divisor $\left\lfloor\frac{1}{2} F-K_{\pi}\right\rfloor=(1,0,1,0,1)$ is not antinef since it has excess -1 at $E_{2}$ and $E_{4}$. The first unloading step is to consider the divisor $\left\lfloor\frac{1}{2} F-K_{\pi}\right\rfloor+E_{2}+$

\footnotetext{
${ }^{7}$ The broken arrows also represent the branches of the strict transform of a curve defined by a generic $f \in \mathfrak{a}$.
} 
$E_{4}=(1,1,1,1,1)$. This divisor has excess -1 at $E_{3}$ and $E_{5}$, so we need to perform a second unloading step to obtain the antinef closure $D_{\lambda_{1}}=(1,1,2,1,2)$.

- The second jumping number is

$$
\begin{aligned}
\lambda_{2} & =\min _{i}\left\{\frac{k_{i}+1+e_{i}^{\lambda_{1}}}{e_{i}}\right\} \\
& =\min _{i}\left\{\frac{2+1}{4}, \frac{3+1}{5}, \frac{5+2}{10}, \frac{3+1}{5}, \frac{5+2}{10}\right\} \\
& =\frac{7}{10} .
\end{aligned}
$$

Then we get $\left\lfloor\frac{7}{10} F-K_{\pi}\right\rfloor=(1,1,3,1,3)$. It has excess -1 at $E_{1}, E_{2}$, and $E_{4}$, and we obtain the divisor $(2,2,3,2,3)$ after the first unloading step. This divisor has excess -1 at $E_{3}$ and $E_{5}$, and, after a second unloading step, we obtain the antinef closure $D_{\lambda_{2}}=(2,2,4,2,4)$.

- The third jumping number is

$$
\begin{aligned}
\lambda_{3} & =\min _{i}\left\{\frac{k_{i}+1+e_{i}^{\lambda_{2}}}{e_{i}}\right\} \\
& =\min _{i}\left\{\frac{2+2}{4}, \frac{3+2}{5}, \frac{5+4}{10}, \frac{3+2}{5}, \frac{5+4}{10}\right\} \\
& =\frac{9}{10} .
\end{aligned}
$$

Then we get $\left\lfloor\frac{9}{10} F-K_{\pi}\right\rfloor=(2,2,5,2,5)$, which has excess -1 at $E_{3}$ and $E_{5}$. After a single unloading step, we get the antinef closure $D_{\lambda_{3}}=(2,3,5,3,5)$.

- The fourth jumping number is

$$
\begin{aligned}
\lambda_{4} & =\min _{i}\left\{\frac{k_{i}+1+e_{i}^{\lambda_{3}}}{e_{i}}\right\} \\
& =\min _{i}\left\{\frac{2+2}{4}, \frac{3+3}{5}, \frac{5+5}{10}, \frac{3+3}{5}, \frac{5+5}{10}\right\} \\
& =1 .
\end{aligned}
$$

Then we get $\left\lfloor F-K_{\pi}\right\rfloor=D_{\lambda_{4}}=(3,3,6,3,6)$ since this divisor is antinef.

- The fifth jumping number is

$$
\begin{aligned}
\lambda_{5} & =\min _{i}\left\{\frac{k_{i}+1+e_{i}^{\lambda_{4}}}{e_{i}}\right\} \\
& =\min _{i}\left\{\frac{2+3}{4}, \frac{3+3}{5}, \frac{5+6}{10}, \frac{3+3}{5}, \frac{5+6}{10}\right\} \\
& =\frac{11}{10} .
\end{aligned}
$$

Then we get $\left\lfloor\frac{11}{10} F-K_{\pi}\right\rfloor=(3,3,7,3,7)$, and, after a single unloading step, we obtain the antinef closure $D_{\lambda_{5}}=(3,4,7,4,7)$. 
Now we will compute the chain of multiplier ideals of the plane curve defined by $f=\left(x^{2}-y^{3}\right)\left(y^{2}-x^{3}\right) \in \mathbb{C}\{x, y\}$. The product of two cusps sharing the origin $O$ is a generic element of the ideal $\mathfrak{a}=\left(x^{2} y^{2}, x^{5}, y^{5}, x y^{4}, x^{4} y\right)$ considered before, so $\mathcal{J}\left(f^{\lambda}\right)=\mathcal{J}\left(\mathfrak{a}^{\lambda}\right)$ for $\lambda<1$. This example will illustrate how the nonexceptional components affect the unloading procedure and, consequently, the list of jumping numbers for $\lambda>1$.

Denote the total transform of the curve defined by $f$ simply as $F$. We represent the relative canonical divisor $K_{\pi}$ and the divisor $F$ in the dual graph as follows:

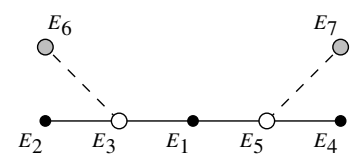

Vertex ordering

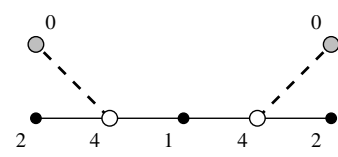

$K_{\pi}$

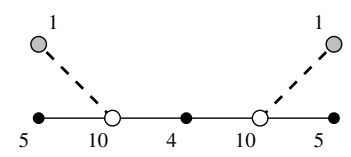

F

The gray dots will represent here the affine components belonging to the strict transform of the curve. The intersection matrix is now

$$
M=\left(\begin{array}{ccccccc}
-5 & 0 & 1 & 0 & 1 & 0 & 0 \\
0 & -2 & 1 & 0 & 0 & 0 & 0 \\
1 & 1 & -1 & 0 & 0 & 1 & 0 \\
0 & 0 & 0 & -2 & 1 & 0 & 0 \\
1 & 0 & 0 & 1 & -1 & 0 & 1
\end{array}\right)
$$

The algorithm is performed as follows:

- The log-canonical threshold is

$$
\lambda_{1}=\operatorname{lct}(\mathfrak{a})=\min _{i}\left\{\frac{k_{i}+1}{e_{i}}\right\}=\min _{i}\left\{\frac{2}{4}, \frac{3}{5}, \frac{5}{10}, \frac{3}{5}, \frac{5}{10}, \frac{1}{1}, \frac{1}{1}\right\}=\frac{1}{2} .
$$

We get $\left\lfloor\frac{1}{2} F-K_{\pi}\right\rfloor=(1,0,1,0,1,0,0)$, and, as in the previous example, its antinef closure is $D_{\lambda_{1}}=(1,1,2,1,2,0,0)$.

- The second jumping number is

$$
\begin{aligned}
\lambda_{2} & =\min _{i}\left\{\frac{k_{i}+1+e_{i}^{\lambda_{1}}}{e_{i}}\right\} \\
& =\min _{i}\left\{\frac{2+1}{4}, \frac{3+1}{5}, \frac{5+2}{10}, \frac{3+1}{5}, \frac{5+2}{10}, \frac{1}{1}, \frac{1}{1}\right\} \\
& =\frac{7}{10} .
\end{aligned}
$$

Then we get $\left\lfloor\frac{7}{10} F-K_{\pi}\right\rfloor=(1,1,3,1,3,0,0)$ and its antinef closure $D_{\lambda_{2}}=$ $(2,2,4,2,4,0,0)$. 
- The third jumping number is

$$
\begin{aligned}
\lambda_{3} & =\min _{i}\left\{\frac{k_{i}+1+e_{i}^{\lambda_{2}}}{e_{i}}\right\} \\
& =\min _{i}\left\{\frac{2+2}{4}, \frac{3+2}{5}, \frac{5+4}{10}, \frac{3+2}{5}, \frac{5+4}{10}, \frac{1}{1}, \frac{1}{1}\right\} \\
& =\frac{9}{10} .
\end{aligned}
$$

Then we get $\left\lfloor\frac{9}{10} F-K_{\pi}\right\rfloor=(2,2,5,2,5,0,0)$ and its antinef closure $D_{\lambda_{3}}=$ $(2,3,5,3,5,0,0)$.

- The fourth jumping number is

$$
\begin{aligned}
\lambda_{4} & =\min _{i}\left\{\frac{k_{i}+1+e_{i}^{\lambda_{3}}}{e_{i}}\right\} \\
& =\min _{i}\left\{\frac{2+2}{4}, \frac{3+3}{5}, \frac{5+5}{10}, \frac{3+3}{5}, \frac{5+5}{10}, \frac{1}{1}, \frac{1}{1}\right\} \\
& =1 .
\end{aligned}
$$

Then we get $\left\lfloor F-K_{\pi}\right\rfloor=(3,3,6,3,6,1,1)$, but this divisor is not antinef because of the nonexceptional components. Namely, we have excess -1 at $E_{3}$ and $E_{5}$. To obtain the antinef closure $D_{\lambda_{4}}=(4,5,10,5,10,1,1)$, we need to perform seven unloading steps with the intermediate divisors:

- $(3,3,7,3,7,1,1)$ with excess -1 at $E_{2}$ and $E_{4}$.

- $(3,4,7,4,7,1,1)$ with excess -1 at $E_{3}$ and $E_{5}$.

- $(3,4,8,4,8,1,1)$ with excess -1 at $E_{1}$.

- $(4,4,8,4,8,1,1)$ with excess -1 at $E_{3}$ and $E_{5}$.

- $(4,4,9,4,9,1,1)$ with excess -1 at $E_{2}$ and $E_{4}$.

- $(4,5,9,5,9,1,1)$ with excess -1 at $E_{3}$ and $E_{5}$.

If we compare with the $\mathfrak{m}$-primary ideal $\mathfrak{a}$, then we should notice that the affine components of $\left\lfloor F-K_{\pi}\right\rfloor$ force us to add more exceptional components when computing its antinef closure, and consequently, this will give a different jumping number in the next step.

- The fifth jumping number is

$$
\begin{aligned}
\lambda_{5} & =\min _{i}\left\{\frac{k_{i}+1+e_{i}^{\lambda_{4}}}{e_{i}}\right\} \\
& =\min _{i}\left\{\frac{2+4}{4}, \frac{3+5}{5}, \frac{5+10}{10}, \frac{3+5}{5}, \frac{5+10}{10}, \frac{2}{1}, \frac{2}{1}\right\} \\
& =\frac{3}{2} .
\end{aligned}
$$

Then we get $\left\lfloor\frac{3}{2} F-K_{\pi}\right\rfloor=(5,5,11,5,11,1,1)$ and its antinef closure $D_{\lambda_{5}}=$ $(5,6,12,6,12,1,1)$. 
Consider a normal surface $X$ with a singularity at $O$. Given a minimal resolution $\pi: X^{\prime} \rightarrow X$ of $X$, Artin [4] introduced the fundamental cycle as the unique smallest nonzero effective divisor with exceptional support that is antinef. Moreover, he proved that the singularity is rational if and only if the arithmetical genus of the fundamental cycle is zero.

We have that $\pi$ is also a minimal log-resolution of the maximal ideal $\mathfrak{m} \subseteq \mathcal{O}_{X, O}$ and the fundamental cycle is the divisor $F$ such that $\mathfrak{m} \cdot \mathcal{O}_{X^{\prime}}=$ $\mathcal{O}_{X^{\prime}}(-F)$. To compute its arithmetical genus, we can use the formula $p_{a}(F)=$ $1+\left(\left(K_{\pi}+F\right) \cdot F\right) / 2$ (see [3]).

This characterization gives us a good source of examples of surfaces with rational singularities.

Example 3.10. Consider a surface $X$ with rational singularity at $O$ whose minimal resolution $\pi: X^{\prime} \rightarrow X$ has six exceptional components $E_{1}, \ldots, E_{6}$ with the following dual graph and intersection matrix:

$$
\left(\begin{array}{cccccc}
-4 & 1 & 1 & 1 & 1 & 1 \\
1 & -5 & 0 & 0 & 0 & 0 \\
1 & 0 & -5 & 0 & 0 & 0 \\
1 & 0 & 0 & -5 & 0 & 0 \\
1 & 0 & 0 & 0 & -5 & 0 \\
1 & 0 & 0 & 0 & 0 & -5
\end{array}\right)
$$

The fundamental cycle is the divisor $F=(2,1,1,1,1,1)$, and the relative canonical divisor is $K_{\pi}=\left(-\frac{5}{3},-\frac{14}{15},-\frac{14}{15},-\frac{14}{15},-\frac{14}{15},-\frac{14}{15}\right)$, so the singularity is not even log-canonical.

The multiplier ideals corresponding to $\lambda_{0}=0$ and $\lambda_{1}=\operatorname{lct}(\mathfrak{m})=\frac{4}{9}$ are given by the antinef divisors $D_{\lambda_{0}}=(2,1,1,1,1,1)$ and $D_{\lambda_{1}}=(3,1,1,1,1,1)$. Notice that $\mathcal{J}\left(\mathfrak{m}^{\lambda_{0}}\right)=\mathfrak{m}$, and, using the techniques of [2], we get that the codimension between these multiplier ideals is 4 .

\subsection{Implementation}

We have implemented Algorithm 3.8 in the computer algebra system Macaulay 2 [12]. The scripts of the source code and the output in full detail of some examples are available at the web page

$$
\text { www.pagines.mal. upc.edu/ jalvz/multiplier.html }
$$

We implemented Tucker's Algorithm 2.14 as well in order to compare both approaches. Of course, once we have the list of jumping numbers, we may use the unloading procedure of Section 2.2 to describe the corresponding multiplier ideals. We have also implemented this extended version of Tucker's algorithm, and it turns out that our method is much faster. 
For example, we have tested the case of an $\mathfrak{m}$-primary ideal $\mathfrak{a}$ whose corresponding dual graph has 35 vertices distributed in three branches only sharing the origin and each branch having three rupture divisors.

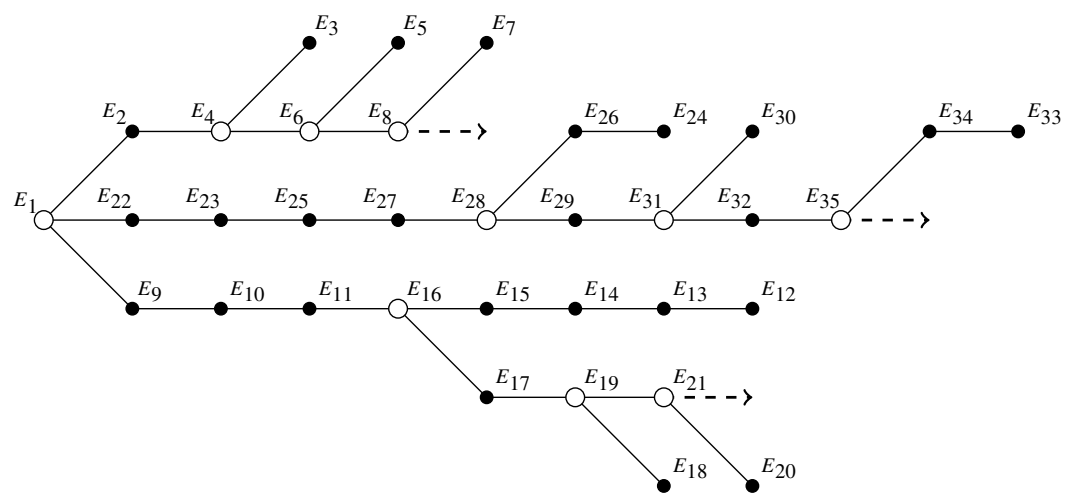

This example has 56,986 jumping numbers in the interval $(0,2]$. Using the extended version of Tucker's algorithm, it takes 897.298 seconds to compute the whole list of jumping numbers and their corresponding multiplier ideals. Using our method, it only takes 372.165 seconds, that is, it is roughly 9 minutes faster.

The main difference between the two algorithms stems in the fact that Tucker needs to find first all the possible critical divisors. We will see in the next section that our algorithm can be understood as a method to find a unique and very precise contributing divisor.

The input that we use in both algorithms, that is, the log-resolution $\pi: X^{\prime} \rightarrow X$ of an ideal $\mathfrak{a} \subseteq \mathcal{O}_{X}$, is encoded using the intersection matrix and the vector of values for the divisor $F$ such that $\mathfrak{a} \cdot \mathcal{O}_{X^{\prime}}=\mathcal{O}_{X^{\prime}}(-F)$. An algorithm to compute this data from a set of generators of the ideal $\mathfrak{a}$ has been described in [1]. An implementation in Macaulay 2 will be available soon. For principal ideals, this can be done using the Singular [13] package alexpoly. lib.

\section{Jumping Divisors}

The theory of critical divisors developed by Tucker [25] focuses on complete ideals very close to a given multiplier ideal. The aim of this section is to understand the whole jump between two consecutive multiplier ideals. For such a purpose, we introduce the following natural definition.

Definition 4.1. Let $\lambda$ be a jumping numbers of an ideal $\mathfrak{a} \subseteq \mathcal{O}_{X, O}$. A reduced divisor $G \leq F$ for which $\lambda$ is a candidate jumping number is called a jumping divisor for $\lambda$ if

$$
\mathcal{J}\left(\mathfrak{a}^{\lambda-\varepsilon}\right)=\pi_{*} \mathcal{O}_{X^{\prime}}\left(\left\lceil K_{\pi}-\lambda F\right\rceil+G\right)
$$

for $\varepsilon$ small enough. We say that a jumping divisor is minimal if no proper subdivisor is a jumping divisor for $\lambda$, that is,

$$
\mathcal{J}\left(\mathfrak{a}^{\lambda-\varepsilon}\right) \nsupseteq \pi_{*} \mathcal{O}_{X^{\prime}}\left(\left\lceil K_{\pi}-\lambda F\right\rceil+G^{\prime}\right)
$$

for any $0 \leq G^{\prime}<G$. 
REMARK 4.2. Any reduced divisor $G \leq F$ for which $\lambda$ is a candidate jumping number defines an ideal nested between two consecutive multiplier ideals

$$
\mathcal{J}\left(\mathfrak{a}^{\lambda-\varepsilon}\right) \supseteq \pi_{*} \mathcal{O}_{X^{\prime}}\left(\left\lceil K_{\pi}-\lambda F\right\rceil+G\right) \supseteq \mathcal{J}\left(\mathfrak{a}^{\lambda}\right) .
$$

Hence, a jumping divisor for $\lambda$ is a contributing divisor to $\lambda$. In particular, a minimal jumping divisor can be understood as the minimal contribution that defines the preceding multiplier ideal.

It is a striking fact that the methods used in the previous section, in particular, our main result, Theorem 3.5, will allow us to construct the unique minimal jumping divisor associated to a jumping number. In fact, we will see in Corollary 4.7 that the only jumping divisors are those reduced divisors $D \leq F$ satisfying $G_{\lambda} \leq D \leq$ $H_{\lambda}$, where $G_{\lambda}$ and $H_{\lambda}$ are defined as follows.

Definition 4.3. Let $\lambda$ be a jumping number of an ideal $\mathfrak{a} \subseteq \mathcal{O}_{X, O}$. Let $D_{\lambda-\varepsilon}=$ $\sum e_{i}^{\lambda-\varepsilon} E_{i}$ be the antinef closure of $\left\lfloor(\lambda-\varepsilon) F-K_{\pi}\right\rfloor$ for $\varepsilon$ small enough. Then we define:

- Maximal jumping divisor is the reduced divisor $H_{\lambda} \leq F$ supported on those components $E_{i}$ for which $\lambda e_{i}-k_{i} \in \mathbb{Z}$. Equivalently,

$$
H_{\lambda}=\left\lceil K_{\pi}-(\lambda-\varepsilon) F\right\rceil-\left\lceil K_{\pi}-\lambda F\right\rceil .
$$

- Minimal jumping divisor is the reduced divisor $G_{\lambda} \leq F$ supported on those components $E_{i}$ for which

$$
\lambda=\frac{k_{i}+1+e_{i}^{\lambda-\varepsilon}}{e_{i}},
$$

that is, supported on those divisors where the minimum considered in Theorem 3.5 is achieved.

It is clear that $H_{\lambda}$ is a jumping divisor and $G_{\lambda} \leq H_{\lambda}$. In fact, any reduced divisor $G \leq F$ that contributes to $\lambda$ satisfies $G \leq H_{\lambda}$. We will prove next that $G_{\lambda}$ deserves the given name.

Proposition 4.4. Let $\lambda$ be a jumping number of an ideal $\mathfrak{a} \subseteq \mathcal{O}_{X, O}$. The reduced divisor $G_{\lambda}$ is a jumping divisor.

Proof. Since $G_{\lambda} \leq H_{\lambda}$, we have $\left\lfloor(\lambda-\varepsilon) F-K_{\pi}\right\rfloor \leq\left\lfloor\lambda F-K_{\pi}\right\rfloor-G_{\lambda}$, and therefore

$$
\mathcal{J}\left(\mathfrak{a}^{\lambda-\varepsilon}\right) \supseteq \pi_{*} \mathcal{O}_{X^{\prime}}\left(\left\lceil K_{\pi}-\lambda F\right\rceil+G_{\lambda}\right) .
$$

For the reverse inclusion, let $D_{\lambda-\varepsilon}=\sum e_{i}^{\lambda-\varepsilon} E_{i}$ be the antinef closure of $\left\lfloor(\lambda-\varepsilon) F-K_{\pi}\right\rfloor$. We want to check that $\left\lfloor\lambda F-K_{\pi}\right\rfloor-G_{\lambda} \leq D_{\lambda-\varepsilon}$. For this purpose, we only need to consider the following cases:

- If $E_{i} \leq G_{\lambda}$, then we have $\lambda=\left(k_{i}+1+e_{i}^{\lambda-\varepsilon}\right) / e_{i}$. In particular, $\left\lfloor\lambda e_{i}-k_{i}\right\rfloor-1=$ $e_{i}^{\lambda-\varepsilon}$.

- If $E_{i} \not \leq G_{\lambda}$, then we have $\lambda<\left(k_{i}+1+e_{i}^{\lambda-\varepsilon}\right) / e_{i}$. Thus, $\left\lfloor\lambda e_{i}-k_{i}\right\rfloor<1+e_{i}^{\lambda-\varepsilon}$ and the result follows. 
The unicity of the jumping divisor $G_{\lambda}$ is a consequence of the following more general statement

THEOREM 4.5. Let $\lambda$ be a jumping number of an ideal $\mathfrak{a} \subseteq \mathcal{O}_{X, O}$. Any contributing divisor $G \leq F$ associated to $\lambda$ satisfies either:

- $\mathcal{J}\left(\mathfrak{a}^{\lambda-\varepsilon}\right)=\pi_{*} \mathcal{O}_{X^{\prime}}\left(\left\lceil K_{\pi}-\lambda F\right\rceil+G\right) \nsupseteq \mathcal{J}\left(\mathfrak{a}^{\lambda}\right)$ if and only if $G_{\lambda} \leq G$, or

- $\mathcal{J}\left(\mathfrak{a}^{\lambda-\varepsilon}\right) \nsupseteq \pi_{*} \mathcal{O}_{X^{\prime}}\left(\left\lceil K_{\pi}-\lambda F\right\rceil+G\right) \nsupseteq \mathcal{J}\left(\mathfrak{a}^{\lambda}\right)$ otherwise.

Proof. Since $G \leq H_{\lambda}$, we have $\left\lfloor(\lambda-\varepsilon) F-K_{\pi}\right\rfloor \leq\left\lfloor\lambda F-K_{\pi}\right\rfloor-G$, and therefore

$$
\mathcal{J}\left(\mathfrak{a}^{\lambda-\varepsilon}\right) \supseteq \pi_{*} \mathcal{O}_{X^{\prime}}\left(\left\lceil K_{\pi}-\lambda F\right\rceil+G\right) .
$$

Now assume that $G_{\lambda} \leq G$. Then $\left\lfloor\lambda F-K_{\pi}\right\rfloor-G \leq\left\lfloor\lambda F-K_{\pi}\right\rfloor-G_{\lambda}$, and using the fact that $G_{\lambda}$ is a jumping divisor, we obtain the equality $\mathcal{J}\left(\mathfrak{a}^{\lambda-\varepsilon}\right)=$ $\pi_{*} \mathcal{O}_{X^{\prime}}\left(\left\lceil K_{\pi}-\lambda F\right\rceil+G\right)$.

If $G_{\lambda} \not \leq G$, then we may consider a component $E_{i} \leq G_{\lambda}$ such that $E_{i} \not \leq G$. Notice that we have

$$
v_{i}\left(D_{\lambda-\varepsilon}\right)=e_{i}^{\lambda-\varepsilon}=\lambda e_{i}-k_{i}-1<\lambda e_{i}-k_{i}=v_{i}\left(\left\lfloor\lambda F-K_{\pi}\right\rfloor-G\right),
$$

where $D_{\lambda-\varepsilon}=\sum e_{i}^{\lambda-\varepsilon} E_{i}$ is the antinef closure of $\left\lfloor(\lambda-\varepsilon) F-K_{\pi}\right\rfloor$. Therefore, by Proposition 3.3 we get the strict inclusion

$$
\mathcal{J}\left(\mathfrak{a}^{\lambda-\varepsilon}\right) \nsupseteq \pi_{*} \mathcal{O}_{X^{\prime}}\left(\left\lceil K_{\pi}-\lambda F\right\rceil+G\right) .
$$

COROLlary 4.6. Let $\lambda$ be a jumping number of an ideal $\mathfrak{a} \subseteq \mathcal{O}_{X, O}$. Then $G_{\lambda}$ is the unique minimal jumping divisor associated to $\lambda$.

Notice that Theorem 4.5 also describes all the jumping divisors associated to a given jumping number. Namely, we have the following:

COROLlary 4.7. Let $\lambda$ be a jumping number of an ideal $\mathfrak{a} \subseteq \mathcal{O}_{X, O}$. Then, any reduced divisor in the interval $G_{\lambda} \leq D \leq H_{\lambda}$ is a jumping divisor.

It is clear from their definition that maximal jumping divisors are periodic, that is, $H_{\lambda}=H_{\lambda+1}$ for any jumping number $\lambda$. On the other hand, critical divisors do not satisfy any periodicity condition. We may find examples where a divisor $G$ is a critical divisor for the jumping number $\lambda$ but not for $\lambda+1$ and vice versa. For minimal jumping divisors we have the following:

Proposition 4.8. Let $\lambda$ be a jumping number of an ideal $\mathfrak{a} \subseteq \mathcal{O}_{X, O}$, and $G_{\lambda}$ its associated minimal jumping divisor. Then we have:

(i) If $\lambda \leq 1$, then $G_{\lambda} \leq G_{\lambda+1}$.

(ii) If $\lambda>1$, then $G_{\lambda}=G_{\lambda+1}$.

Proof. Assume that there exists a prime divisor $E_{i} \leq G_{\lambda}$ such that $E_{i} \not \leq G_{\lambda+1}$. Then, for a sufficiently small $\varepsilon>0$, we have

$$
\lambda=\frac{k_{i}+1+e_{i}^{\lambda-\varepsilon}}{e_{i}} \text { and } \lambda+1<\frac{k_{i}+1+e_{i}^{(\lambda-\varepsilon)+1}}{e_{i}},
$$


where $D_{\lambda-\varepsilon}=\sum e_{i}^{\lambda-\varepsilon} E_{i}$ denotes the antinef closure of $\left\lfloor(\lambda-\varepsilon) F-K_{\pi}\right\rfloor$, and, equivalently, $D_{(\lambda-\varepsilon)+1}=\sum e_{i}^{(\lambda-\varepsilon)+1} E_{i}$ is the antinef closure of $\lfloor((\lambda-\varepsilon)+1) \times$ $\left.F-K_{\pi}\right\rfloor$.

Therefore,

$$
\frac{k_{i}+1+e_{i}^{\lambda-\varepsilon}}{e_{i}}+1<\frac{k_{i}+1+e_{i}^{(\lambda-\varepsilon)+1}}{e_{i}}
$$

or, equivalently, $e_{i}^{\lambda-\varepsilon}+e_{i}<e_{i}^{(\lambda-\varepsilon)+1}$. Then we have $\mathfrak{a} \cdot \mathcal{J}\left(\mathfrak{a}^{\lambda-\varepsilon}\right) \nsubseteq \mathcal{J}\left(\mathfrak{a}^{(\lambda-\varepsilon)+1}\right)$, so we get a contradiction.

For $\lambda>1$, we have the equality $e_{i}^{\lambda-\varepsilon}+e_{i}=e_{i}^{(\lambda-\varepsilon)+1}$ because of Skoda's theorem, so the result follows.

Let $\lambda^{\prime}<\lambda$ be two consecutive jumping numbers of an ideal $\mathfrak{a} \subseteq \mathcal{O}_{X, O}$. It is quite surprising that the minimal jumping divisor $G_{\lambda}$ gives such a nice approach to the understanding of the jump from $\mathcal{J}\left(\mathfrak{a}^{\lambda}\right)$ to its preceding multiplier ideal $\mathcal{J}\left(\mathfrak{a}^{\lambda^{\prime}}\right)$. Taking into account that its construction is based on Theorem 3.5, where $\lambda$ is obtained from the antinef divisor associated to $\mathcal{J}\left(\mathfrak{a}^{\lambda^{\prime}}\right)$, it would seem more natural to consider the jump in the other direction. It turns out that the jump from $\mathcal{J}\left(\mathfrak{a}^{\lambda^{\prime}}\right)$ to $\mathcal{J}\left(\mathfrak{a}^{\lambda}\right)$ does not behave that nicely.

Proposition 4.9. Let $\lambda^{\prime}<\lambda$ be two consecutive jumping numbers of an ideal $\mathfrak{a} \subseteq \mathcal{O}_{X, O}$, and $D_{\lambda^{\prime}}$ be the antinef closure of $\left\lfloor\lambda^{\prime} F-K_{\pi}\right\rfloor$. Then we have:

(i) $\mathcal{J}\left(\mathfrak{a}^{\lambda^{\prime}}\right) \nsupseteq \pi_{*} \mathcal{O}_{X^{\prime}}\left(-D_{\lambda^{\prime}}-G_{\lambda}\right)=\mathcal{J}\left(\mathfrak{a}^{\lambda}\right)$.

(ii) $\mathcal{J}\left(\mathfrak{a}^{\lambda^{\prime}}\right) \nsupseteq \pi_{*} \mathcal{O}_{X^{\prime}}\left(\left\lceil K_{\pi}-(\lambda-\varepsilon) F\right\rceil-G_{\lambda}\right)=\mathcal{J}\left(\mathfrak{a}^{\lambda}\right)$.

Proof. Let $D_{\lambda^{\prime}}=\sum e_{i}^{\lambda^{\prime}} E_{i}, D_{\lambda}=\sum e_{i}^{\lambda} E_{i}$ be the antinef closures of $\left\lfloor\lambda^{\prime} F-K_{\pi}\right\rfloor$ and $\left\lfloor\lambda F-K_{\pi}\right\rfloor$, respectively.

(i) Since $G_{\lambda}$ is a jumping divisor, we have $\left\lfloor\lambda F-K_{\pi}\right\rfloor-G_{\lambda} \leq D_{\lambda^{\prime}}$, and hence $\left\lfloor\lambda F-K_{\pi}\right\rfloor \leq D_{\lambda^{\prime}}+G_{\lambda}$. This gives the inclusion $\pi_{*} \mathcal{O}_{X^{\prime}}\left(-D_{\lambda^{\prime}}-G_{\lambda}\right) \subseteq \mathcal{J}\left(\mathfrak{a}^{\lambda}\right)$.

In order to check the reverse inclusion $\pi_{*} \mathcal{O}_{X^{\prime}}\left(-D_{\lambda^{\prime}}-G_{\lambda}\right) \supseteq \mathcal{J}\left(\mathfrak{a}^{\lambda}\right)$, it is enough, using Proposition 3.3, to prove $v_{i}\left(D_{\lambda^{\prime}}+G_{\lambda}\right) \leq v_{i}\left(D_{\lambda}\right)=e_{i}^{\lambda}$ for any component $E_{i}$. We have $e_{i}^{\lambda^{\prime}} \leq e_{i}^{\lambda}$ just because $\mathcal{J}\left(\mathfrak{a}^{\lambda^{\prime}}\right) \nsupseteq \mathcal{J}\left(\mathfrak{a}^{\lambda}\right)$, and the inequality is strict when $E_{i} \leq G_{\lambda}$, so the result follows.

(ii) Let $D^{\prime}$ be the antinef closure of $\left\lfloor(\lambda-\varepsilon) F-K_{\pi}\right\rfloor+G_{\lambda}$. Since $G_{\lambda} \leq H_{\lambda}$, we have

$$
\left\lfloor(\lambda-\varepsilon) F-K_{\pi}\right\rfloor+G_{\lambda} \leq\left\lfloor\lambda F-K_{\pi}\right\rfloor \leq D_{\lambda},
$$

so we have the inclusion $\pi_{*} \mathcal{O}_{X^{\prime}}\left(\left\lceil K_{\pi}-(\lambda-\varepsilon) F\right\rceil-G_{\lambda}\right) \supseteq \mathcal{J}\left(\mathfrak{a}^{\lambda}\right)$. In order to prove the reverse inclusion, we introduce the auxiliary divisor $D=\sum d_{i} E_{i} \in \Lambda$ defined as follows:

- $d_{i}=\left\lfloor(\lambda-\varepsilon) e_{i}-k_{i}\right\rfloor+1$ if $E_{i} \leq G_{\lambda}$,

- $d_{i}=e_{i}^{\lambda^{\prime}}$ if $E_{i} \leq H_{\lambda}$ but $E_{i} \not \leq G_{\lambda}$,

- $d_{i}=\left\lfloor(\lambda-\varepsilon) e_{i}-k_{i}\right\rfloor$ otherwise.

Clearly, we have $\left\lfloor(\lambda-\varepsilon) F-K_{\pi}\right\rfloor+G_{\lambda} \leq D$, but we also have $\left\lfloor\lambda F-K_{\pi}\right\rfloor \leq$ D. Indeed, 
- For $E_{i} \leq G_{\lambda}$, we have $\left\lfloor\lambda e_{i}-k_{i}\right\rfloor=\lambda e_{i}-k_{i}=\left\lfloor(\lambda-\varepsilon) e_{i}-k_{i}\right\rfloor+1=d_{i}$.

- If $\lambda$ is a candidate for $E_{i}$ but $E_{i} \not \leq G_{\lambda}$, then $\left\lfloor\lambda e_{i}-k_{i}\right\rfloor=\lambda e_{i}-k_{i}<1+e_{i}^{\lambda^{\prime}}$, and hence $\left\lfloor\lambda e_{i}-k_{i}\right\rfloor \leq e_{i}^{\lambda^{\prime}}=d_{i}$.

- Otherwise, $\left\lfloor\lambda e_{i}-k_{i}\right\rfloor=\left\lfloor(\lambda-\varepsilon) e_{i}-k_{i}\right\rfloor=d_{i}$.

Therefore, taking antinef closures, we have $D^{\prime} \leq D_{\lambda} \leq \widetilde{D}$. On the other hand, $D \leq D^{\prime}$. Namely, $v_{i}\left(D^{\prime}\right) \geq e_{i}^{\lambda^{\prime}}$ at any $E_{i}$ because $\left\lfloor\lambda^{\prime} F-K_{\pi}\right\rfloor \leq\lfloor(\lambda-\varepsilon) F-$ $\left.K_{\pi}\right\rfloor+G_{\lambda}$. Moreover, $v_{i}\left(D^{\prime}\right) \geq\left\lfloor(\lambda-\varepsilon) e_{i}-k_{i}\right\rfloor+\delta_{i}^{G_{\lambda}}$ by the definition of antinef closure. Here, $\delta_{i}^{G_{\lambda}}=1$ if $E_{i} \leq G_{\lambda}$ and zero otherwise. Thus, $v_{i}\left(D^{\prime}\right) \geq v_{i}(D)$, as desired. As a consequence, $\widetilde{D} \leq D^{\prime}$, which, together with the previous $D^{\prime} \leq D_{\lambda} \leq$ $\widetilde{D}$, gives $\widetilde{D}=D^{\prime}=D_{\lambda}$, and the result follows.

REMARK 4.10. Contrary to the case of Theorem $4.5, G_{\lambda}$ may not be minimal. In fact, we will see in Example 5.8 a divisor $G<G_{\lambda}$ satisfying:

$$
\mathcal{J}\left(\mathfrak{a}^{\lambda^{\prime}}\right)=\pi_{*} \mathcal{O}_{X^{\prime}}\left(-D_{\lambda^{\prime}}\right) \nsupseteq \pi_{*} \mathcal{O}_{X^{\prime}}\left(-D_{\lambda^{\prime}}-G\right)=\mathcal{J}\left(\mathfrak{a}^{\lambda}\right) .
$$

Despite the fact that the antinef closure of both $\left\lfloor(\lambda-\varepsilon) F-K_{\pi}\right\rfloor$ and $\left\lfloor\lambda^{\prime} F-K_{\pi}\right\rfloor$ is $D_{\lambda^{\prime}}$, it is quite remarkable that the above jumping property does not hold taking $\left\lfloor\lambda^{\prime} F-K_{\pi}\right\rfloor$, that is, the equality $\pi_{*} \mathcal{O}_{X^{\prime}}\left(\left\lfloor\lambda^{\prime} F-K_{\pi}\right\rfloor-G_{\lambda}\right)=\mathcal{J}\left(\mathfrak{a}^{\lambda}\right)$ is not always true.

\subsection{Invariance of the Minimal Jumping Divisor with Respect to the Log-Resolution}

Multiplier ideals and jumping numbers are known to be independent of the chosen log-resolution of the initial ideal $\mathfrak{a} \subseteq \mathcal{O}_{X, O}$. The aim of this section is to prove that the minimal jumping divisor is generically independent of the log-resolution in a sense that we will make precise further. As a consequence of Proposition 5.6 and Corollary 5.5 in Section 5, critical divisors will also be generically independent of the log-resolution. This is a remarkable fact since, as it was pointed out by Tucker in [25, Rem. 3.4], there is no reason to believe that critical divisors (and by extension minimal jumping divisors) are independent of the resolution since they depend on all the divisorial valuations appearing in $F$.

We start fixing some notation that we will use in this section. Let $\pi^{\prime}: X^{\prime} \rightarrow X$ be the minimal log-resolution of an ideal $\mathfrak{a} \subseteq \mathcal{O}_{X, O}$. Any other log-resolution $\pi: Y \rightarrow X$ factors through $\pi^{\prime}$, that is, there is a birational morphism $g: Y \rightarrow X^{\prime}$ such that $\pi=\pi^{\prime} \circ g$ (see [19, Thm. 4.1]).

For a given jumping number $\lambda$ of $\mathfrak{a}$, we will denote $G_{\lambda}^{\prime}$ the minimal jumping divisor of $\pi^{\prime}$ and $E_{1}^{\prime}, \ldots, E_{r}^{\prime}$ the exceptional components of $E^{\prime}=\operatorname{Exc}\left(\pi^{\prime}\right)$. If $G_{\lambda}$ and $E_{1}, \ldots, E_{s}$ are the minimal jumping divisor and the exceptional components of $E=\operatorname{Exc}(\pi)$ for any other $\log$-resolution $\pi$, then we will enumerate them setting $E_{i}$ equal to the strict transform by $g$ of $E_{i}^{\prime}$ for $1 \leq i \leq r$. If no confusion arise, then we will use the same symbol to denote a divisor $D=\sum_{i=1}^{r} d_{i} E_{i}^{\prime}$ on $X^{\prime}$ or its strict transform $D=\sum_{i=1}^{r} d_{i} E_{i}$ on $Y$. 
THEOREM 4.11. With the previous notation, $G_{\lambda}$ is independent of the logresolution $\pi$ if and only if $\pi$ does not include any blowing-up at points in the intersection of two components of the minimal jumping divisor $G_{\lambda}^{\prime}$ of the minimal log-resolution.

In fact, from the proof of this result we can express the minimal jumping divisor of any resolution. For such a purpose, we need to fix some notation.

A reduced divisor with exceptional support $D=E_{i_{1}}+\cdots+E_{i_{m}} \leq E$ is a chain with ends $E_{i_{1}}$ and $E_{i_{m}}$ if $a_{D}\left(E_{i_{1}}\right)=a_{D}\left(E_{i_{m}}\right)=1$ and $a_{D}\left(E_{i_{k}}\right)=2$ for any other $1<k<m$. Given $E_{j_{1}}, E_{j_{2}} \leq E$, we say that this chain connects $E_{j_{1}}$ and $E_{j_{2}}$ if $E_{j_{1}} \in \operatorname{Adj}\left(E_{i_{1}}\right)$ and $E_{j_{2}} \in \operatorname{Adj}\left(E_{i_{m}}\right)$. Observe that if $E_{j_{1}}$ and $E_{j_{2}}$ are adjacent in $E$, then $D=0$ is a chain connecting them.

Corollary 4.12. We have

$$
G_{\lambda}=G_{\lambda}^{\prime}+\sum_{\substack{E_{i}^{\prime}+E_{j}^{\prime} \leq G_{\lambda}^{\prime} \\ E_{i}^{\prime} \cdot E_{j}^{\prime}=1}} D_{i j},
$$

where $D_{i j}$ is a chain connecting $E_{i}$ and $E_{j}$.

Consider generic log-resolutions as those obtained from a minimal one by further blowing-ups at simple (and hence generic) points on the exceptional components. Then, Theorem 4.11 states that generic log-resolutions have the same minimal jumping divisor. This genericity may be formulated, when $X$ is smooth, in terms of valuations in the valuative tree $\mathcal{V}$ of Favre and Jonsson [8]. Consider the dual graphs $\Gamma$ and $\Gamma^{\prime}$ of $E$ and $E^{\prime}$, respectively, embedded in the valuative tree $\mathcal{V}$ as in [8, Chap. 6], and let $v_{i}$ denote the divisorial valuation centered at $E_{i}$.

COROllary 4.13. The minimal jumping divisor $G_{\lambda}$ of $\pi$ equals the minimal jumping divisor $G_{\lambda}^{\prime}$ if and only if $\Gamma$ has no vertex inside any segment $] v_{i}, v_{j}[$ for which $E_{i}^{\prime}$ and $E_{j}^{\prime}$ are adjacent in $E^{\prime}$ and belong to $G_{\lambda}^{\prime}$.

Proof of Theorem 4.11. Let $\lambda^{\prime}<\lambda$ be two consecutive jumping numbers of $\mathfrak{a}$. We will argue by induction on the number of blowing-ups needed to reach $Y$ from a minimal resolution. In order to simplify the notation, we will assume in this proof that $X^{\prime}$ also dominates a minimal log-resolution and that $Y$ is obtained from $X^{\prime}$ by one blowing-up $g: Y \rightarrow X^{\prime}$ at a closed point $p \in X^{\prime}$ giving the exceptional component $E_{s}$. Assume that (4.1) holds on $X^{\prime}$ and let us prove it on $Y$. Notice that, keeping the notation used in this section, we are in the case $r+1=s$.

Let $F^{\prime}=\sum_{i=1}^{r} e_{i} E_{i}^{\prime}$ and $F=\sum_{i=1}^{s} e_{i} E_{i}$ be the divisors in $X^{\prime}$ and $Y$, respectively, such that $\mathfrak{a} \mathcal{O}_{X^{\prime}}=\mathcal{O}_{X^{\prime}}\left(-F^{\prime}\right)$ and $\mathfrak{a} \mathcal{O}_{Y}=\mathcal{O}_{Y}(-F)$. We also consider the antinef divisors $D_{\lambda^{\prime}}^{\prime}=\sum_{i=1}^{r} e_{i}^{\lambda^{\prime}} E_{i}^{\prime}$ and $D_{\lambda^{\prime}}=\sum_{i=1}^{s} e_{i}^{\lambda^{\prime}} E_{i}$ for which $\mathcal{J}\left(\mathfrak{a}^{\lambda^{\prime}}\right)=\pi_{*}^{\prime} \mathcal{O}_{X^{\prime}}\left(-D_{\lambda^{\prime}}^{\prime}\right)=\pi_{*} \mathcal{O}_{Y}\left(-D_{\lambda^{\prime}}\right)$ sharing the first $r$ coefficients since 
multiplier ideals are independent of the log-resolution. Moreover, by Theorem 3.5,

$$
\lambda=\min _{1 \leq i \leq r}\left\{\frac{k_{i}+1+e_{i}^{\lambda^{\prime}}}{e_{i}}\right\}=\min _{1 \leq i \leq s}\left\{\frac{k_{i}+1+e_{i}^{\lambda^{\prime}}}{e_{i}}\right\},
$$

clearly demonstrating that the strict transform of $G_{\lambda}^{\prime}$ is contained in $G_{\lambda}$. In particular, $\lambda e_{i}-k_{i}=1+e_{i}^{\lambda^{\prime}}$ if and only if $E_{i} \leq G_{\lambda}$ and $\lambda e_{i}-k_{i}<1+e_{i}^{\lambda^{\prime}}$ otherwise.

We distinguish two cases:

(i) The closed point $p$ lies only on one exceptional divisor $E_{j}^{\prime}$. Then we have $e_{s}=e_{j}, k_{s}=k_{j}+1$, and $e_{s}^{\lambda^{\prime}}=e_{j}^{\lambda^{\prime}}$, and thus

$$
v_{s}\left(\left\lfloor\lambda F-K_{\pi}\right\rfloor\right)=\left\lfloor\lambda e_{s}-k_{i}\right\rfloor=\left\lfloor\lambda e_{j}-k_{j}\right\rfloor-1 \leq e_{j}^{\lambda^{\prime}}=e_{s}^{\lambda^{\prime}} .
$$

Hence, $E_{s}$ cannot belong to $G_{\lambda}$.

(ii) The closed point $p$ lies on the intersection of two exceptional divisors $E_{j_{1}}^{\prime}$ and $E_{j_{2}}^{\prime}$. Then we have $e_{s}=e_{j_{1}}+e_{j_{2}}, k_{s}=k_{j_{1}}+k_{j_{2}}+1$, and $e_{s}^{\lambda^{\prime}}=e_{j_{1}}^{\lambda^{\prime}}+e_{j_{2}}^{\lambda^{\prime}}$, so

$$
\begin{aligned}
v_{s}\left(\left\lfloor\lambda F-K_{\pi}\right\rfloor\right) & =\left\lfloor\lambda e_{s}-k_{s}\right\rfloor \\
& =\left\lfloor\lambda e_{j_{1}}-k_{j_{1}}+\lambda e_{j_{2}}-k_{j_{2}}\right\rfloor-1 \leq e_{j_{1}}^{\lambda^{\prime}}+e_{j_{2}}^{\lambda^{\prime}}+1=e_{s}^{\lambda^{\prime}}+1,
\end{aligned}
$$

and equality holds if and only if $E_{j_{1}}^{\prime}+E_{j_{2}}^{\prime} \leq G_{\lambda}$. In particular, $E_{s}$ does not belong to $G_{\lambda}$ whenever none or just one of the components $E_{j_{1}}^{\prime}, E_{j_{2}}^{\prime}$ belong to $G_{\lambda}^{\prime}$.

\subsection{Geometric Properties of Minimal Jumping Divisors in the Dual Graph}

Assume that a critical divisor $G$ associated to a jumping number $\lambda$ has exceptional support. One of the key ingredients in Tucker's algorithm for the computation of jumping numbers is that $G$ satisfies some nice geometric conditions when viewed in the dual graph: $G$ is a connected chain, and its ends must be either rupture or dicritical divisors (see Proposition 2.13). Then, it is natural to ask whether jumping divisors satisfy analogous properties.

In this section we also assume that the minimal jumping divisor $G_{\lambda}$ has exceptional support. Then, it may have several connected components in the dual graph, and these components are not necessarily chains. However, we can still control the ends of each component. To prove the main result of this section (see Theorem 4.17), we need some preliminary results. Keep the notation of Section 2.

Lemma 4.14. Let $\lambda$ be a jumping number of an ideal $\mathfrak{a} \subseteq \mathcal{O}_{X, O}$. For any component $E_{i}$ of the minimal jumping divisor $G_{\lambda}$, we have

$$
\left(\left\lceil K_{\pi}-\lambda F\right\rceil+G_{\lambda}\right) \cdot E_{i}=-2+\lambda \rho_{i}+\sum_{E_{j} \in \operatorname{Adj}\left(E_{i}\right)}\left\{\lambda e_{j}-k_{j}\right\}+a_{G_{\lambda}}\left(E_{i}\right) .
$$


Proof. For any $E_{i} \leq G_{\lambda}$, we have

$$
\begin{aligned}
\left(\left\lceilK_{\pi}\right.\right. & \left.-\lambda F\rceil+G_{\lambda}\right) \cdot E_{i} \\
& =\left(\left(K_{\pi}-\lambda F\right)+\left\{-K_{\pi}+\lambda F\right\}+G_{\lambda}-E_{i}+E_{i}\right) \cdot E_{i} \\
& =\left(K_{\pi}+E_{i}\right) \cdot E_{i}-\lambda F \cdot E_{i}+\left\{\lambda F-K_{\pi}\right\} \cdot E_{i}+\left(G_{\lambda}-E_{i}\right) \cdot E_{i} .
\end{aligned}
$$

Let us now compute each summand separately. First, the adjunction formula gives $\left(K_{\pi}+E_{i}\right) \cdot E_{i}=-2$ because $E_{i} \cong \mathbb{P}^{1}$. As for the second and fourth terms, the equality $-\lambda F \cdot E_{i}=\lambda \rho_{i}$ follows from the definition of the excesses, and clearly $a_{G_{\lambda}}\left(E_{i}\right)=\left(G_{\lambda}-E_{i}\right) \cdot E_{i}$ because $E_{i} \leq G_{\lambda}$.

Therefore, it only remains to prove that

$$
\left\{\lambda F-K_{\pi}\right\} \cdot E_{i}=\sum_{E_{j} \in \operatorname{Adj}\left(E_{i}\right)}\left\{\lambda e_{j}-k_{j}\right\},
$$

which is also quite immediate. Indeed, writing

$$
\left\{\lambda F-K_{\pi}\right\}=\sum_{j=1}^{r}\left\{\lambda e_{j}-k_{j}\right\} E_{j},
$$

equality (4.2) follows by observing that (for $j \neq i$ ), $E_{j} \cdot E_{i}=1$ if and only if $E_{j} \in \operatorname{Adj}\left(E_{i}\right)$, and the term corresponding to $j=i$ vanishes because we have $\lambda e_{i}-k_{i} \in \mathbb{Z}$.

REMARK 4.15. It is important to notice that $\left(\left\lceil K_{\pi}-\lambda F\right\rceil+G_{\lambda}\right) \cdot E_{i} \in \mathbb{Z}$, that is, $-2+\sum_{E_{j} \in \operatorname{Adj}\left(E_{i}\right)}\left\{\lambda e_{j}-k_{j}\right\}+\lambda \rho_{i}+a_{G_{\lambda}}\left(E_{i}\right) \in \mathbb{Z}$.

The following result is an analogue of the numerical conditions that critical divisors satisfy (see Proposition 4.19). Unfortunately, it does not provide a characterization of minimal jumping divisors.

Proposition 4.16. Let $\lambda$ be a jumping number of an ideal $\mathfrak{a} \subseteq \mathcal{O}_{X, O}$. For any component $E_{i} \leq G_{\lambda}$ of the minimal jumping divisor $G_{\lambda}$, we have

$$
\left(\left\lceil K_{\pi}-\lambda F\right\rceil+G_{\lambda}\right) \cdot E_{i} \geq 0 .
$$

Proof. Let $G_{\lambda}$ be the minimal jumping divisor. Given a prime divisor $E_{i} \leq G_{\lambda}$, we consider the short exact sequence

$$
\begin{aligned}
0 \longrightarrow \mathcal{O}_{X^{\prime}}\left(\left\lceil K_{\pi}-\lambda F\right\rceil+G_{\lambda}-E_{i}\right) \longrightarrow \mathcal{O}_{X^{\prime}}\left(\left\lceil K_{\pi}-\lambda F\right\rceil+G_{\lambda}\right) \\
\quad \longrightarrow \mathcal{O}_{E_{i}}\left(\left\lceil K_{\pi}-\lambda F\right\rceil+G_{\lambda}\right) \longrightarrow 0 .
\end{aligned}
$$

Pushing it forward to $X$, we get

$$
\begin{aligned}
0 & \longrightarrow \pi_{*} \mathcal{O}_{X^{\prime}}\left(\left\lceil K_{\pi}-\lambda F\right\rceil+G_{\lambda}-E_{i}\right) \longrightarrow \pi_{*} \mathcal{O}_{X^{\prime}}\left(\left\lceil K_{\pi}-\lambda F\right\rceil+G_{\lambda}\right) \\
& \longrightarrow H^{0}\left(E_{i}, \mathcal{O}_{E_{i}}\left(\left\lceil K_{\pi}-\lambda F\right\rceil+G_{\lambda}\right)\right) \otimes \mathbb{C}_{O},
\end{aligned}
$$

where $\mathbb{C}_{O}$ denotes the skyscraper sheaf supported at $O$ with fiber $\mathbb{C}$. The minimality of $G_{\lambda}$ (see Theorem 4.5) implies that

$$
\pi_{*} \mathcal{O}_{X^{\prime}}\left(\left\lceil K_{\pi}-\lambda F\right\rceil+G_{\lambda}-E_{i}\right) \neq \pi_{*} \mathcal{O}_{X^{\prime}}\left(\left\lceil K_{\pi}-\lambda F\right\rceil+G_{\lambda}\right) .
$$


Thus, $H^{0}\left(E_{i}, \mathcal{O}_{E_{i}}\left(\left\lceil K_{\pi}-\lambda F\right\rceil+G_{\lambda}\right)\right) \neq 0$ or, equivalently, $\left(\left\lceil K_{\pi}-\lambda F\right\rceil+G_{\lambda}\right)$. $E_{i} \geq 0$.

With these ingredients, we can provide the following geometric property of minimal jumping divisors when viewed in the dual graph.

THEOREM 4.17. Let $G_{\lambda}$ be the minimal jumping divisor associated to a jumping number $\lambda$ of an ideal $\mathfrak{a} \subseteq \mathcal{O}_{X, O}$. Then the ends of a connected component of $G_{\lambda}$ must be either rupture or dicritical divisors.

Proof. Assume that an end $E_{i}$ of a connected component of $G_{\lambda}$ is neither a rupture nor a dicritical divisor. This means that $E_{i}$ has no excess, that is, $\rho_{i}=0$, and that it has one or two adjacent divisors, say $E_{j}$ and $E_{l}$, in the dual graph, but at most one of them belongs to $G_{\lambda}$.

For the case that $E_{i}$ has two adjacent divisors $E_{j}$ and $E_{l}$, the formula given in Lemma 4.14 reduces to $\left(\left\lceil K_{\pi}-\lambda F\right\rceil+G_{\lambda}\right) \cdot E_{i}=-2+\left\{\lambda e_{j}-k_{j}\right\}+\left\{\lambda e_{l}-k_{l}\right\}+$ $\lambda \rho_{i}+a_{G_{\lambda}}\left(E_{i}\right)$. Then:

- If $E_{i}$ has valence one in $G_{\lambda}$, for example, $E_{l} \not \leq G_{\lambda}$, then

$$
\left(\left\lceil K_{\pi}-\lambda F\right\rceil+G_{\lambda}\right) \cdot E_{i}=-2+\left\{\lambda e_{l}-k_{l}\right\}+1<0 .
$$

- If $E_{i}$ is an isolated component of $G_{\lambda}$, that is, $E_{j}, E_{l} \not \leq G_{\lambda}$, then

$$
\left(\left\lceil K_{\pi}-\lambda F\right\rceil+G_{\lambda}\right) \cdot E_{i}=-2+\left\{\lambda e_{j}-k_{j}\right\}+\left\{\lambda e_{l}-k_{l}\right\}<0 .
$$

If $E_{i}$ has just one adjacent divisor $E_{j}$, that is, $E_{i}$ is an end of the dual graph, the formula reduces to $\left(\left\lceil K_{\pi}-\lambda F\right\rceil+G_{\lambda}\right) \cdot E_{i}=-2+\left\{\lambda e_{j}-k_{j}\right\}+\lambda \rho_{i}+a_{G_{\lambda}}\left(E_{i}\right)$. Then:

- If $E_{i}$ has valence one in $G_{\lambda}$, then $\left(\left\lceil K_{\pi}-\lambda F\right\rceil+G_{\lambda}\right) \cdot E_{i}=-2+1<0$.

- If $E_{i}$ is an isolated component of $G_{\lambda}$, then

$$
\left(\left\lceil K_{\pi}-\lambda F\right\rceil+G_{\lambda}\right) \cdot E_{i}=-2+\left\{\lambda e_{j}-k_{j}\right\}<0 .
$$

In any case we get a contradiction with Proposition 4.16.

REMARK 4.18. It follows from [26, Thm. 3.3] that the minimal jumping divisor associated to the log-canonical threshold is connected in the case that $X$ is smooth.

As a consequence, we may also give the following refinement of Proposition 4.16.

Proposition 4.19. Let $\lambda$ be a jumping number of an $\mathfrak{m}$-primary ideal $\mathfrak{a} \subseteq \mathcal{O}_{X, O}$. If $E_{i} \leq G_{\lambda}$ is neither a rupture nor a dicritical component of the minimal jumping divisor $G_{\lambda}$, then we have

$$
\left(\left\lceil K_{\pi}-\lambda F\right\rceil+G_{\lambda}\right) \cdot E_{i}=0 .
$$

Proof. Assume that $E_{i} \leq G_{\lambda}$ is neither a rupture or a dicritical component. In particular, it is not the end of a connected component of $G_{\lambda}$. Thus, $E_{i}$ has exactly two adjacent components $E_{j}$ and $E_{l}$ in $G_{\lambda}$, and its excess is $\rho_{i}=0$. The formula given in Lemma 4.14 reduces to

$$
\left(\left\lceil K_{\pi}-\lambda F\right\rceil+G_{\lambda}\right) \cdot E_{i}=-2+\lambda \rho_{i}+\left\{\lambda e_{j}-k_{j}\right\}+\left\{\lambda e_{l}-k_{l}\right\}+a_{G_{\lambda}}\left(E_{i}\right) .
$$


Notice that $a_{G_{\lambda}}\left(E_{i}\right)=2$ and also $\left\{\lambda e_{j}-k_{j}\right\}=\left\{\lambda e_{l}-k_{l}\right\}=0$ because $E_{j}$ and $E_{l}$ are components of $G_{\lambda}$, so finally $\left(\left\lceil K_{\pi}-\lambda F\right\rceil+G_{\lambda}\right) \cdot E_{i}=0$.

\section{Minimal Contributing Divisors}

The theory of minimal jumping divisors introduced in Section 4 can be included in a more general framework that we will describe in this section. For such a purpose, we will give our own perspective of the work of Hyry and Järviletho [14] and its relation with the theory of contributing divisors of Tucker [25].

Let $\lambda$ be a jumping number of an ideal $\mathfrak{a} \subseteq \mathcal{O}_{X, O}$. Recall that a reduced divisor $G \leq F$ that contributes to $\lambda$ defines an ideal nested between two consecutive multiplier ideals

$$
\mathcal{J}\left(\mathfrak{a}^{\lambda-\varepsilon}\right) \supseteq \pi_{*} \mathcal{O}_{X^{\prime}}\left(\left\lceil K_{\pi}-\lambda F\right\rceil+G\right) \supseteq \mathcal{J}\left(\mathfrak{a}^{\lambda}\right) .
$$

We may interpret that $\lambda$ is parameterized by the set of nested ideals defined by contributions, but this is far from being a one-to-one correspondence. An easy way to detect such a nested ideal is finding a suitable critical divisor using Tucker's algorithm. The approach given in the previous sections is more economical in the sense that each jumping number is parameterized by its unique minimal jumping divisor $G_{\lambda}$ or, equivalently, by its preceding multiplier ideal.

Hyry and Järviletho [14] give a similar approach where jumping numbers are parameterized by general antinef divisors ${ }^{8}$ or, equivalently, by complete ideals that are not necessarily nested in the chain of multiplier ideals. We should point out that their results also hold for the case that $X$ has rational singularities since their arguments are based on divisorial considerations. Given any antinef divisor $D=\sum d_{i} E_{i} \in \operatorname{Div}\left(X^{\prime}\right)$, they considered the following notions:

- Jumping number corresponding to $D$,

$$
\lambda_{D}:=\min _{i}\left\{\frac{k_{i}+1+d_{i}}{e_{i}}\right\} .
$$

- Support of a jumping number corresponding to D,

$$
S_{D}:=\left\{i \mid \lambda_{D}=\frac{k_{i}+1+d_{i}}{e_{i}}\right\} .
$$

- Contributing divisor associated to D,

$$
G_{D}:=\sum_{i \in S_{D}} E_{i}
$$

Hyry and Järviletho proved in [14, Prop. 1] that all jumping numbers of $\mathfrak{a}$ can be obtained as $\lambda_{D}$ for a suitable antinef divisor $D \in \operatorname{Div}\left(X^{\prime}\right)$ (or, equivalently, a complete ideal $I_{D}$ ). Moreover, they give in [14, Thm. 1] a combinatorial criterion that detects the existence of such antinef divisors. The simplest parameterizations they used to describe the set of jumping numbers are given by antinef divisors corresponding to critical divisors (see [14, Thm. 2]).

\footnotetext{
${ }^{8}$ Hyry and Järviletho only consider the case of $\mathfrak{m}$-primary ideals on smooth surfaces and, consequently, antinef divisors with exceptional support, but their ideas also hold in general.
} 
In general, the complete ideal $I_{D}$ associated to an antinef divisor $D \in \operatorname{Div}\left(X^{\prime}\right)$ satisfies $\mathcal{J}\left(\mathfrak{a}^{\lambda_{D}-\varepsilon}\right) \supseteq I_{D}$ but does not necessarily contain $\mathcal{J}\left(\mathfrak{a}^{\lambda}\right)$. However, if $I_{D}$ is nested in between two consecutive multiplier ideals

$$
\mathcal{J}\left(\mathfrak{a}^{\lambda-\varepsilon}\right) \supseteq I_{D} \supseteq \mathcal{J}\left(\mathfrak{a}^{\lambda}\right),
$$

then it must satisfy $\lambda=\lambda_{D}$.

REMARK 5.1. We can also interpret this framework through the generalized version of log-canonical thresholds already introduced by Järviletho [15]. Namely, the log-canonical threshold with respect to any other ideal $\mathfrak{b} \subseteq \mathcal{O}_{X, O}$ is defined as

$$
\operatorname{lct}_{\mathfrak{b}}(\mathfrak{a}):=\inf \left\{c \in \mathbb{Q}_{>0} \mid \mathcal{J}\left(\mathfrak{a}^{c}\right) \not \supset \mathfrak{b}\right\} .
$$

Notice that whenever $I_{D}$ is the complete ideal associated to an antinef divisor $D \in \operatorname{Div}\left(X^{\prime}\right)$, then $\lambda_{D}=\operatorname{lct}_{I_{D}}(\mathfrak{a})$.

Hyry and Järviletho [14, Lemma 11] proved that if $D \in \operatorname{Div}\left(X^{\prime}\right)$ is an antinef divisor, then $G_{D}$ is a contributing divisor for $\lambda_{D}$. In fact, the contributing divisors obtained in this way satisfy some nice properties as we will see next.

Proposition 5.2. Let $G$ be a contributing divisor associated to a jumping number $\lambda$. Let $D$ be the antinef closure of $\left\lfloor\lambda F-K_{\pi}\right\rfloor-G$. Then $G_{D} \leq G$.

Proof. Let $D=\sum d_{i} E_{i}$ be the antinef closure of $\left\lfloor\lambda F-K_{\pi}\right\rfloor-G$. Since $I_{D}$ is a nested ideal in the chain of multiplier ideals, then we have

$$
\lambda=\lambda_{D}=\min _{i}\left\{\frac{k_{i}+1+d_{i}}{e_{i}}\right\} .
$$

Hence, $\lambda e_{i}-k_{i} \leq 1+d_{i}$, and equality holds if and only if $i \in S_{D}$. In order to prove $G_{D} \leq G$, we will show that $E_{i} \not \leq G$ implies $E_{i} \not \leq G_{D}$. Indeed, if $E_{i} \not \leq G$ and $E_{i} \leq G_{D}$, then $\left\lfloor\lambda e_{i}-k_{i}\right\rfloor \leq d_{i}$ (just because $\left\lfloor\lambda F-K_{\pi}\right\rfloor-G \leq D$ by Lemma 2.3) and $\lambda e_{i}-k_{i}-1=d_{i}$, so we get a contradiction.

Proposition 5.3. Let $\lambda=\lambda_{D^{\prime}}$ be a jumping number associated to an antinef divisor $D^{\prime} \in \operatorname{Div}\left(X^{\prime}\right)$. Let $D$ be the antinef closure of $\left\lfloor\lambda F-K_{\pi}\right\rfloor-G_{D^{\prime}}$. Then we have $D \leq D^{\prime}, \lambda_{D}=\lambda_{D^{\prime}}, S_{D}=S_{D^{\prime}}$, and $G_{D}=G_{D^{\prime}}$.

Proof. Using the definition of antinef closure (see Lemma 2.3), in order to get $D \leq D^{\prime}$, we only need to prove that $\left\lfloor\lambda F-K_{\pi}\right\rfloor-G_{D^{\prime}} \leq D^{\prime}$. Set $D^{\prime}=\sum d_{i}^{\prime} E_{i}$. By hypothesis,

$$
\lambda=\lambda_{D^{\prime}}=\min _{i}\left\{\frac{k_{i}+1+d_{i}^{\prime}}{e_{i}}\right\} .
$$

Therefore, we have $\left\lfloor\lambda e_{i}-k_{i}\right\rfloor \leq d_{i}^{\prime}$ if $i \notin S_{D^{\prime}}$, whereas $\left\lfloor\lambda e_{i}-k_{i}\right\rfloor-1=d_{i}^{\prime}$ if $i \in S_{D^{\prime}}$, as desired.

Notice then that we have $\mathcal{J}\left(\mathfrak{a}^{\lambda-\varepsilon}\right) \supseteq I_{D} \supseteq I_{D^{\prime}}$, so, given the fact that $I_{D^{\prime}} \nsubseteq$ $\mathcal{J}\left(\mathfrak{a}^{\lambda}\right)$, we get $\lambda_{D}=\lambda$. Now, the inclusion of divisors $D \leq D^{\prime}$ having the same minimum $\lambda_{D}=\lambda_{D^{\prime}}$ gives the inclusion of supports $S_{D} \supseteq S_{D^{\prime}}$ and, equivalently, 
$G_{D} \geq G_{D^{\prime}}$. On the other hand, taking $G=G_{D^{\prime}}$ in Proposition 5.2, we get the reverse inequality of divisors $G_{D} \leq G_{D^{\prime}}$, so we are done.

The main result of this section is that we can find a minimal contributing divisor among all contributing divisors defining the same nested ideal.

THEOREM 5.4. Let $G$ be a contributing divisor associated to a jumping number $\lambda$. Let $D$ be the antinef closure of $\left\lfloor\lambda F-K_{\pi}\right\rfloor-G$, which gives a nested ideal

$$
\mathcal{J}\left(\mathfrak{a}^{\lambda-\varepsilon}\right) \supseteq I_{D}=\pi_{*} \mathcal{O}_{X^{\prime}}\left(\left\lceil K_{\pi}-\lambda F\right\rceil+G\right) \supseteq \mathcal{J}\left(\mathfrak{a}^{\lambda}\right) .
$$

Then we also have $I_{D}=\pi_{*} \mathcal{O}_{X^{\prime}}\left(\left\lceil K_{\pi}-\lambda F\right\rceil+G_{D}\right)$. Furthermore, $G_{D}$ is the minimal contributing divisor associated to $\lambda$ that defines the same ideal $I_{D}$, that is:

- Any contribution $G^{\prime}$ to $\lambda$ defining $I_{D}=\pi_{*} \mathcal{O}_{X^{\prime}}\left(\left\lceil K_{\pi}-\lambda F\right\rceil+G^{\prime}\right)$ must satisfy $G_{D} \leq G^{\prime}$.

- Any proper subdivisor $G^{\prime}<G_{D}$ defines a strictly included ideal

$$
I_{D} \supseteq \pi_{*} \mathcal{O}_{X^{\prime}}\left(\left\lceil K_{\pi}-\lambda F\right\rceil+G^{\prime}\right) .
$$

Proof. Let $D^{\prime}$ be the antinef closure of $\left\lfloor\lambda F-K_{\pi}\right\rfloor-G_{D}$. We will first see that $D=D^{\prime}$, thus giving the desired equality of ideals

$$
I_{D}=\pi_{*} \mathcal{O}_{X^{\prime}}\left(\left\lceil K_{\pi}-\lambda F\right\rceil+G\right)=\pi_{*} \mathcal{O}_{X^{\prime}}\left(\left\lceil K_{\pi}-\lambda F\right\rceil+G_{D}\right)=I_{D^{\prime}} .
$$

By Proposition 5.2 we have $G_{D} \leq G$, so $\left\lfloor\lambda F-K_{\pi}\right\rfloor-G \leq\left\lfloor\lambda F-K_{\pi}\right\rfloor-G_{D}$ and $D \leq D^{\prime}$. The reverse inequality $D \geq D^{\prime}$ is a consequence of Proposition 5.3.

To show that $G_{D}$ is the minimal contributor to the jumping number $\lambda$ that defines the same ideal $I_{D}$, we will prove the following equivalent result.

CLAIM. Any contributor $G^{\prime}$ to $\lambda$ for which $I_{D} \supseteq \pi_{*} \mathcal{O}_{X^{\prime}}\left(\left\lceil K_{\pi}-\lambda F\right\rceil+G^{\prime}\right)$ also satisfies the reverse inclusion $I_{D} \subseteq \pi_{*} \mathcal{O}_{X^{\prime}}\left(\left\lceil K_{\pi}-\lambda F\right\rceil+G^{\prime}\right)$ if and only if $G_{D} \leq G^{\prime}$.

Proof. Suppose first that $G_{D} \leq G^{\prime}$. Then $\left\lfloor\lambda F-K_{\pi}\right\rfloor-G^{\prime} \leq\left\lfloor\lambda F-K_{\pi}\right\rfloor-G_{D}$, and hence $D^{\prime \prime} \leq D^{\prime}=D$, where $D^{\prime \prime}$ is the antinef closure of $\left\lfloor\lambda F-K_{\pi}\right\rfloor-G^{\prime}$. Therefore, $I_{D} \subseteq I_{D^{\prime \prime}}$, as wanted.

Assume now that $G_{D} \not \leq G^{\prime}$ and pick a component $E_{i} \leq G_{D}$ such that $E_{i} \not \leq G^{\prime}$. By hypothesis, $I_{D} \supseteq I_{D^{\prime \prime}}$ and, equivalently, $D \leq D^{\prime \prime}$, but in fact $D<D^{\prime \prime}$ since

$$
v_{i}(D)=\lambda e_{i}-k_{i}-1<\lambda e_{i}-k_{i}=v_{i}\left(\left\lfloor\lambda F-K_{\pi}\right\rfloor-G^{\prime}\right) \leq v_{i}\left(D^{\prime \prime}\right) .
$$

The result then follows from Proposition 3.3.

It turns out that critical divisors are also minimal as we can see in the following generalization of [14, Prop. 3].

Corollary 5.5. Let $G$ be a contributing divisor associated to a jumping number $\lambda$. Let $D$ be the antinef closure of $\left\lfloor\lambda F-K_{\pi}\right\rfloor-G$. Then $G$ is a critical divisor if and only if $G_{D}=G$ and $I_{D}$ and $\mathcal{J}\left(\mathfrak{a}^{\lambda}\right)$ do not admit strictly nested ideals between them defined by contributors to $\lambda$. 
Proof. Assume first that $G_{D}=G$. Then, by Theorem 5.4, any proper subdivisor $0 \leq G^{\prime}<G$ defines an ideal strictly included in $I_{D} \supseteq \pi_{*} \mathcal{O}_{X^{\prime}}\left(\left\lceil K_{\pi}-\lambda F\right\rceil+G^{\prime}\right) \supseteq$ $\mathcal{J}\left(\mathfrak{a}^{\lambda}\right)$. Since $I_{D}$ and $\mathcal{J}\left(\mathfrak{a}^{\lambda}\right)$ do not admit strictly nested ideals between them coming from contributors, we get $\pi_{*} \mathcal{O}_{X^{\prime}}\left(\left\lceil K_{\pi}-\lambda F\right\rceil+G^{\prime}\right)=\mathcal{J}\left(\mathfrak{a}^{\lambda}\right)$, so $G$ is a critical divisor.

Assume now that $G$ is a critical divisor. By Proposition 5.2 we have $G_{D} \leq$ $G$. Both divisors define the same ideal by Theorem 5.4, so they must be equal; otherwise, we would have a contradiction with the fact that $G$ is a critical divisor.

Finally, we will see that there is no contributing divisor $G^{\prime}$ associated to $\lambda$ defining a strictly nested ideal

$$
I_{D} \supseteq \pi_{*} \mathcal{O}_{X^{\prime}}\left(\left\lceil K_{\pi}-\lambda F\right\rceil+G^{\prime}\right) \nsupseteq \mathcal{J}\left(\mathfrak{a}^{\lambda}\right) .
$$

Assume that such $G^{\prime}$ exists and let $D^{\prime}$ be the antinef closure of $\lfloor\lambda F-$ $\left.K_{\pi}\right\rfloor-G^{\prime}$. Then the inclusion of divisors $D<D^{\prime}$ having the same minimum $\lambda_{D}=\lambda_{D^{\prime}}=\lambda$ implies $S_{D^{\prime}} \subseteq S_{D}$ and $G_{D^{\prime}} \leq G_{D}$. Since $G=G_{D}$ is minimal, applying Theorem 5.4, we must have $G=G_{D}=G_{D^{\prime}} \leq G^{\prime}$, contradicting the starting hypothesis of inclusion of ideals.

The minimal jumping divisor introduced in Section 4 fits nicely in this theory. Given a jumping number $\lambda$ of an m-primary ideal $\mathfrak{a} \subseteq \mathcal{O}_{X, O}$, let $D_{\lambda-\varepsilon}$ be the antinef closure of $\left\lfloor(\lambda-\varepsilon) F-K_{\pi}\right\rfloor$ for $\varepsilon>0$ small enough. Then we have $\lambda=$ $\lambda_{D_{\lambda-\varepsilon}}$, and the unique minimal jumping divisor is $G_{\lambda}=G_{D_{\lambda-\varepsilon}}$.

In general, a divisor $G \in \Lambda$ that contributes to the jumping number $\lambda$ might not be contained in $G_{\lambda}$. For minimal contributing divisors, we have the following:

Proposition 5.6. Let $\lambda$ be a jumping number of an ideal $\mathfrak{a} \subseteq \mathcal{O}_{X, O}$, and $G_{\lambda}$ be its associated minimal jumping divisor. Then $G_{D} \leq G_{\lambda}$ for any antinef divisor $D \in \operatorname{Div}\left(X^{\prime}\right)$ such that $\lambda=\lambda_{D}$.

Proof. Let $D^{\prime}$ be the antinef closure of $\left\lfloor\lambda F-K_{\pi}\right\rfloor-G_{D}$. By Proposition 5.3 we have $G_{D}=G_{D^{\prime}}$ and $\lambda=\lambda_{D}=\lambda_{D^{\prime}}$. Since the ideals $\mathcal{J}\left(\mathfrak{a}^{\lambda-\varepsilon}\right) \supseteq I_{D^{\prime}}$ are nested, their corresponding antinef divisors satisfy $D_{\lambda-\varepsilon} \leq D^{\prime}$, and they reach the same minimum $\lambda_{D_{\lambda-\varepsilon}}=\lambda_{D^{\prime}}=\lambda$. Hence, $S_{D^{\prime}} \subseteq S_{D_{\lambda-\varepsilon}}$, which implies $G_{D}=G_{D^{\prime}} \leq$ $G_{\lambda}$, as we wanted.

COROLlary 5.7. Let $\lambda$ be a jumping number of an ideal $\mathfrak{a} \subseteq \mathcal{O}_{X, O}$. Then we have $G \leq G_{\lambda}$ for any critical divisor $G$ associated to $\lambda$.

The reduced sum of all critical divisors equals the jumping divisor $G_{\lambda}$ for simple complete ideals (see [11, Thm. 2.3] for the smooth case). However, this is no longer true in general.

EXAmple 5.8. Let $X$ be a smooth surface and consider the $\mathfrak{m}$-primary ideal $\mathfrak{a} \subseteq$ $\mathcal{O}_{X, O}$ whose dual graph is

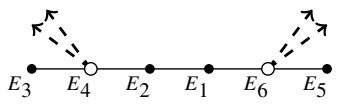

Vertex ordering

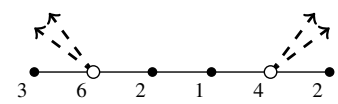

$K_{\pi}$

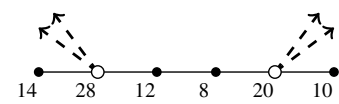

F 
The multiplier ideals corresponding to the consecutive jumping numbers $\frac{5}{7}<\frac{3}{4}$ are
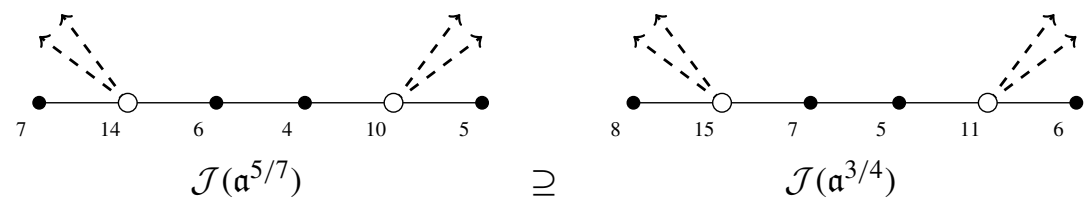

The minimal jumping divisor corresponding to $\lambda=\frac{3}{4}$ is $G_{3 / 4}=E_{1}+E_{2}+$ $E_{4}+E_{6}$, but the only critical divisors are $E_{4}$ and $E_{6}$. In particular,

$$
\mathcal{J}\left(\mathfrak{a}^{5 / 7}\right) \nsupseteq \pi_{*} \mathcal{O}_{X^{\prime}}\left(\left\lceil K_{\pi}-\frac{3}{4} F\right\rceil+E_{4}+E_{6}\right) .
$$

It is worth pointing out that

$$
\pi_{*} \mathcal{O}_{X^{\prime}}\left(-D_{5 / 7}-E_{4}-E_{6}\right)=\pi_{*} \mathcal{O}_{X^{\prime}}\left(-D_{5 / 7}-G_{3 / 4}\right)=\mathcal{J}\left(\mathfrak{a}^{3 / 4}\right),
$$

where $D_{5 / 7}$ is the antinef closure of $\left\lfloor\frac{5}{7} F-K_{\pi}\right\rfloor$. So minimality is not always achieved for the divisor $G_{\lambda}$ in Proposition 4.9.

In general, not every nested ideal between two consecutive multiplier ideals is given by a contributing divisor. The following result identifies them precisely.

Proposition 5.9. Any nested ideal $\mathcal{J}\left(\mathfrak{a}^{\lambda-\varepsilon}\right) \supseteq I_{D^{\prime}} \supseteq \mathcal{J}\left(\mathfrak{a}^{\lambda}\right)$ comes from a contributing divisor $G$ associated to $\lambda$, that is, $I_{D^{\prime}}=\pi_{*} \mathcal{O}_{X^{\prime}}\left(\left\lceil K_{\pi}-\lambda F\right\rceil+G\right)$, if and only if $D^{\prime}=D$ where $D$ is the antinef closure of $\left\lfloor\lambda F-K_{\pi}\right\rfloor-G$, and in this case $G=G_{D^{\prime}}$.

Proof. Let $D^{\prime}$ be the antinef closure of $\left\lfloor\lambda F-K_{\pi}\right\rfloor-G$. By Proposition 5.3 we have $D \leq D^{\prime}$. On the other hand, Proposition 5.2 implies $G_{D^{\prime}} \leq G$, which gives

$$
\left\lfloor\lambda F-K_{\pi}\right\rfloor-G \leq\left\lfloor\lambda F-K_{\pi}\right\rfloor-G_{D^{\prime}},
$$

and hence $D^{\prime} \leq D$, so we get the desired result. The reverse implication is straightforward.

Proposition 5.10. Let $I_{D}$ be the ideal associated to an antinef divisor $D \in \Lambda$. Then, $I_{D}$ is a multiplier ideal for the ideal $\mathfrak{a} \subseteq \mathcal{O}_{X, O}$ if and only if $D$ is contained in the antinef closure of $\left\lfloor\left(\lambda_{D}-\varepsilon\right) F-K_{\pi}\right\rfloor$. If this is the case, then $D$ is also the antinef closure of $\left\lfloor\lambda_{D} F-K_{\pi}\right\rfloor-G_{D}$.

Proof. By definition we have $\left\lfloor\left(\lambda_{D}-\varepsilon\right) F-K_{\pi}\right\rfloor \leq D$ because $\mathcal{J}\left(\mathfrak{a}^{\lambda_{D}-\varepsilon}\right) \supseteq I_{D}$. We also have $I_{D} \nsubseteq \mathcal{J}\left(\mathfrak{a}^{\lambda}\right)$, so the only possibility for $I_{D}$ of being a multiplier ideal is when $\mathcal{J}\left(\mathfrak{a}^{\lambda} D^{-\varepsilon}\right)=I_{D}$, so, applying Lemma 3.1, $D$ must be contained in the antinef closure of $\left\lfloor\left(\lambda_{D}-\varepsilon\right) F-K_{\pi}\right\rfloor$. The rest of the statement follows from Theorem 5.4. 
AcKnowledgments. We wish to thank Víctor González Alonso for uncountable discussions that we had with him during the realization of this work. The authors would also like to thank Pierrette Cassou-Nogués and Wim Veys for the comments received and Manuel González-Villa for a careful reading of a previous version of the manuscript.

\section{References}

[1] M. Alberich-Carramiñana, J. Àlvarez Montaner, and G. Blanco, Effective computation of base points of two-dimensional ideals, preprint.

[2] M. Alberich-Carramiñana, J. Àlvarez Montaner, F. Dachs-Cadefau, and V. GonzálezAlonso, Poincaré series of multiplier ideals in two-dimensional local rings with rational singularities, preprint, arXiv:1412.3607.

[3] M. Artin, Some numerical criteria for contractability of curves on algebraic surfaces, Amer. J. Math. 84 (1962), 485-496.

[4] - On isolated rational singularities of surfaces, Amer. J. Math. 88 (1966), 129-136.

[5] E. Casas-Alvero, Singularities of plane curves, London Math. Soc. Lecture Note Ser., 276, Cambridge University Press, Cambridge, 2000.

[6] L. Ein, R. Lazarsfeld, K. Smith, and D. Varolin, Jumping coefficients of multiplier ideals, Duke Math. J. 123 (2004), 469-506.

[7] F. Enriques and O. Chisini, Lezioni sulla teoria geometrca delle equazioni e delle funzioni algebriche, N. Zanichelli, Bologna, 1915.

[8] C. Favre and M. Jonsson, The valuative tree, Lecture Notes in Math., 1853, SpringerVerlag, Berlin, 2004.

[9] _ Valuations and multiplier ideals, J. Amer. Math. Soc. 18 (2005), 655-684.

[10] C. Galindo, F. Hernando, and F. Monserrat, The log-canonical threshold of a plane curve, Math. Proc. Cambridge Math. Soc. 160 (2016), 513-535.

[11] C. Galindo and F. Monserrat, The Poincaré series of multiplier ideals of a simple complete ideal in a local ring of a smooth surface, Adv. Math. 225 (2010), 10461068.

[12] D. Grayson and M. Stillman, Macaulay 2, 〈http://www.math.uiuc.edu/Macaulay2〉.

[13] G.-M. Greuel, G. Pfister, and H. Schönemann, Singular 2.0.5: A computer algebra system for polynomial computations, Centre for Computer Algebra, University of Kaiserslautern, 2003, 〈http://www.singular.uni-kl.de〉.

[14] E. Hyry and T. Järviletho, Jumping numbers and ordered tree structures on the dual graph, Manuscripta Math. 136 (2011), 411-437.

[15] T. Järviletho, Jumping numbers of a simple complete ideal in a two-dimensional regular local ring, Mem. Amer. Math. Soc. 214 (2011), no. 1009.

[16] T. Kuwata, On log canonical thresholds of reducible plane curves, Amer. J. Math. 121 (1999), 701-721.

[17] H. Laufer, On rational singularities, Amer. J. Math. 94 (1972), 597-608.

[18] R. Lazarsfeld, Positivity in algebraic geometry II, 49, Springer-Verlag, Berlin, 2004.

[19] J. Lipman, Rational singularities, with applications to algebraic surfaces and unique factorization, Inst. Hautes Études Sci. Publ. Math. 36 (1969), 195-279.

[20] J. Lipman and K. I. Watanabe, Integrally closed ideals in two-dimensional regular local rings are multiplier ideals, Math. Res. Lett. 10 (2003), 423-434.

[21] D. Naie, Jumping numbers of a unibranch curve on a smooth surface, Manuscripta Math. 128 (2009), 33-49. 
[22] A. J. Reguera, Curves and proximity on rational surface singularities, J. Pure Appl. Algebra 122 (1997), 107-126.

[23] K. E. Smith and H. Thompson, Irrelevant exceptional divisors for curves on a smooth surface, Algebra, geometry and their interactions, Contemp. Math., 448, pp. 245254, Amer. Math. Soc., Providence, RI, 2007.

[24] K. Tucker, Integrally closed ideals on log terminal surfaces are multiplier ideals, Math. Res. Lett. 16 (2009), 903-908.

[25] _ Jumping numbers on algebraic surfaces with rational singularities, Trans. Amer. Math. Soc. 362 (2010), 3223-3241.

[26] W. Veys, Determination of the poles of the topological zeta function for curves, Manuscripta Math. 87 (1995), 435-448.

M. Alberich-Carramiñana

Departament de Matemàtiques

Universitat Politècnica de Catalunya

Av. Diagonal 647

Barcelona 08028

Spain

Maria.Alberich@upc.edu

F. Dachs-Cadefau

Department of Mathematics

KU Leuven

Celestijnenlaan 200B box 2400

BE-3001 Leuven

Belgium

Ferran.DachsCadefau@wis.kuleuven.be
J. Àlvarez Montaner

Departament de Matemàtiques

Universitat Politècnica de Catalunya

Av. Diagonal 647

Barcelona 08028

Spain

Josep.Alvarez@upc.edu 